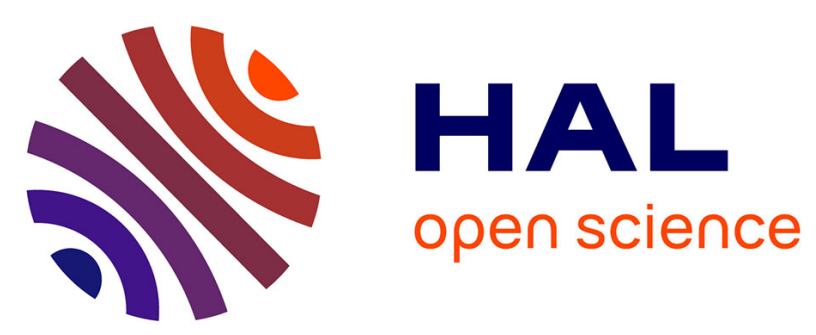

\title{
Drop spreading at the impact in the Leidenfrost boiling
} Guillaume Castanet, Ophélie Caballina, Fabrice Lemoine

\section{To cite this version:}

Guillaume Castanet, Ophélie Caballina, Fabrice Lemoine. Drop spreading at the impact in the Leidenfrost boiling. Physics of Fluids, 2015, 27 (6), pp.063302. 10.1063/1.4922066 . hal-01499164

\section{HAL Id: hal-01499164 \\ https://hal.science/hal-01499164}

Submitted on 31 Mar 2017

HAL is a multi-disciplinary open access archive for the deposit and dissemination of scientific research documents, whether they are published or not. The documents may come from teaching and research institutions in France or abroad, or from public or private research centers.
L'archive ouverte pluridisciplinaire HAL, est destinée au dépôt et à la diffusion de documents scientifiques de niveau recherche, publiés ou non, émanant des établissements d'enseignement et de recherche français ou étrangers, des laboratoires publics ou privés.

\section{(ㅇ)(1) $\$$}

Distributed under a Creative Commons Attribution - NonCommercial - NoDerivatives| 4.0 


\title{
Drop spreading at the impact in the Leidenfrost boiling
}

\author{
G. Castanet, ${ }^{1,2, a)}$ O. Caballina,,$^{1,2}$ and F. Lemoine ${ }^{1,2}$ \\ ${ }^{1}$ LEMTA, UMR 7563, Université de Lorraine, Vandoeuvre-Lès-Nancy 54518, France \\ ${ }^{2}$ LEMTA, UMR 7563, CNRS, Vandoeuvre-Lès-Nancy 54518, France
}

\begin{abstract}
Although the Leidenfrost effect has been extensively studied in the past, one challenge for the modeling of this phenomenon remains, namely, how to determine the effect induced by the presence of a vapor film on the frictions exerted on the drop. To address this issue, experiments are carried out on liquids with very different viscosities including water, ethanol, and several mixtures of water and glycerol. The deformation of droplets of a few hundred micrometers, impinging a perfectly smooth solid surface heated above the Leidenfrost temperature, is observed by shadowgraphy using a high-speed camera. Experimental results are compared to a theoretical model which is based on an inviscid asymptotic solution for the flow inside the lamella. This model also considers a lamella thickness which does not depend on the viscosity, the surface tension, and thus on the Reynolds and Weber numbers. This description of the lamella is valid if Weber and Reynolds numbers are high enough. Mass and momentum balances applied to the rim bounding the spreading lamella yield an equation for the rim motion which is then solved numerically. This equation accounts for the momentum transferred to the rim by the liquid coming from the lamella, the capillary forces, and the viscous stress at the separation between the lamella and the rim. The comparison between the model and the experiments suggests that the liquid at the bottom edge of the lamella is dragged by the vapor film given that the vapor velocity in the vapor film is significantly larger than that of the liquid. This process significantly increases the drop spreading for the low viscosity liquids. An analysis of the viscous boundary layer which develops at the bottom edge of the lamella is found to confirm this scenario.
\end{abstract}

\section{INTRODUCTION}

The impact of droplets on superheated surfaces is central to many technological processes. In combustion engines, the impact of fuel droplets is responsible for the deposition and build-up of liquid films in the manifold of gasoline engines ${ }^{1}$ which usually result in the inhomogeneous and incomplete combustion of the fuel and the emission of pollutants. Drop interactions with superheated surfaces are also encountered in the spray cooling of hot metals in the steel industry. An ideal quench would generally process at a very fast rate to avoid the precipitation of the alloy constituents and thus produce alloys of better strength and hardness. However, quenching begins in the film boiling regime, at wall temperatures well above the Leidenfrost temperature. An insulating film of vapor develops at the interface between the liquid and the solid surface. The absence of direct contact between the wall and the liquid coolant is highly detrimental to the heat transfers. ${ }^{2}$ The control and optimization of such processes remain challenging given current incomplete knowledge of the complex flow and heat transfers associated with the drop/wall interaction, especially in Leidenfrost conditions. The outcome of an impact is influenced by many parameters related to the impact conditions, liquid and air properties, and the nature of the substrate and its topology. Two main dimensionless parameters are usually used to describe the impact conditions: the Weber number $W e=\rho U_{0}^{2} d_{0} / \sigma$ and the Reynolds number $R e=U_{0} d_{0} / v$, where $U_{0}$ and $d_{0}$ are the impact velocity and the initial droplet diameter, respectively, 
$\nu, \rho$, and $\sigma$ are, respectively, the kinematic viscosity, density, and surface tension of the liquid. The Ohnesorge number, $O h$, is also frequently introduced but this is a combination of the Reynolds and Weber numbers $\left(O h=W e^{1 / 2} R e^{-1}\right)$. Different evolutions have been reported in the literature depending on the impact conditions and these have included drop deposition, partial or complete rebound, prompt splash, corona splash, and receding breakup. ${ }^{3}$ These outcomes are also influenced by whether the collided surface is smooth, rough, and porous, dry or wetted. In the film boiling regime, the non-wetting of the hot surface reduces the possibilities to rebound and splashing. ${ }^{4,5}$ At low Weber numbers, drops spread radially after impact, possibly form a lamella bounded by a torus rim, reach a maximum spreading diameter $d_{x, \max }$, and then recoil before bouncing without any disintegration. At higher Weber numbers, the onset of droplet disintegration is characterized by the formation of a small number of secondary droplets, usually one to three satellite drops (partial rebound). Above a critical Weber number (typically $W e>80$ for water), the drop breaks up into numerous smaller droplets (splashing regime). Previous works have studied the main characteristics of the dynamic of a droplet impinging a hot wall in the Leidenfrost conditions: the spreading diameter, ${ }^{4,6,7}$ the loss of momentum, ${ }^{4,8}$ the contact time of the drop near the wall, ${ }^{6,8}$ the spreading time, ${ }^{6,9}$ the onset of the splashing, ${ }^{10}$ and the dynamic Leidenfrost temperature. ${ }^{11,12}$ All these characteristic properties have been shown to be function of the Weber number, but for some, the exact nature of this function is still a matter of debate. Unlike the impact on isothermal solid surface, ${ }^{13,14}$ the influence of the liquid viscosity has not yet been subject of thorough investigation in the film boiling regime. Most of the data reported in the literature concern water and ethanol,,${ }^{4,7}$ which have rather low and similar viscosities but different surface tensions.

Basically, the spreading behavior of liquid droplets impinging on isothermal surface is affected by the wall friction and by the forces associated with wettability. These forces are not relevant in the Leidenfrost regime, and the drop can be intuitively expected to spread over a larger area. This was recently confirmed by comparing the spreading diameter in the case of an impact on a superhydrophobic substrate and in Leidenfrost conditions. ${ }^{7,15}$ It was suggested that the vapor released in the Leidenfrost effect could drag the liquid and thus lead to higher drop spreading. According to Tran et al. ${ }^{16}$ maximum spreading occurs when the shear stress at the interface with the vapor film balances the capillary force. Based on that assumption, they were able to theoretically determine that $d_{x, \max } / d_{0} \sim W e^{3 / 10}$, but this scaling does not fully match with the experiments which instead suggested $d_{x, \max } / d_{0} \sim W e^{0.39}$ for $W e>10$.

In the past, approaches based on the balance of mechanical energies have been frequently used to model droplet spreading. They attempted to incorporate the viscous dissipation by making certain basic assumptions about the flow field inside the drop. ${ }^{17-19}$ Also, in most of these models, the droplet shape is approximated by a disc of uniform thickness, even though the shape of the drop is never similar to that of a flat disc. Usually, no liquid motion is considered at the time of maximum spread whereas in fact there are rotational velocities in the rim that develop at the edge of the spreading lamella. Recent studies ${ }^{20-23}$ indicate that the liquid motion inside the lamella can be described well by an axisymmetric inviscid flow with a radial velocity $u_{r} \sim r / t$. Consequently, viscous dissipation would not occur inside the lamella where the motion is nearly conservative but rather in the rim where there is basically a rotational flow and at the bottom edge of the lamella where frictions are exerted on the droplet.

The present study is an experimental investigation of the spreading of droplets in Leidenfrost conditions. Shadow imaging with a high-speed camera is used to record the deformation of obliquely impacting droplets. Some of the main drop evolution characteristics such as the maximum spreading diameter can be determined from the image processing. Also, mixtures of water and glycerol offer the possibility of covering a wide range of impact conditions. Measurements are analyzed on the basis of a theoretical approach initiated by Roisman et al. to describe the spreading of droplets in the impact on a wetting surface ${ }^{24,25}$ or in the binary collisions. ${ }^{26}$

\section{EXPERIMENTAL SETUP AND MEASUREMENT TECHNIQUES}

The experimental setup used to study the dynamic of the droplets in the Leidenfrost regime is shown in Fig. 1. A piezoelectric injector is used to produce a chain of mono-sized and equally 


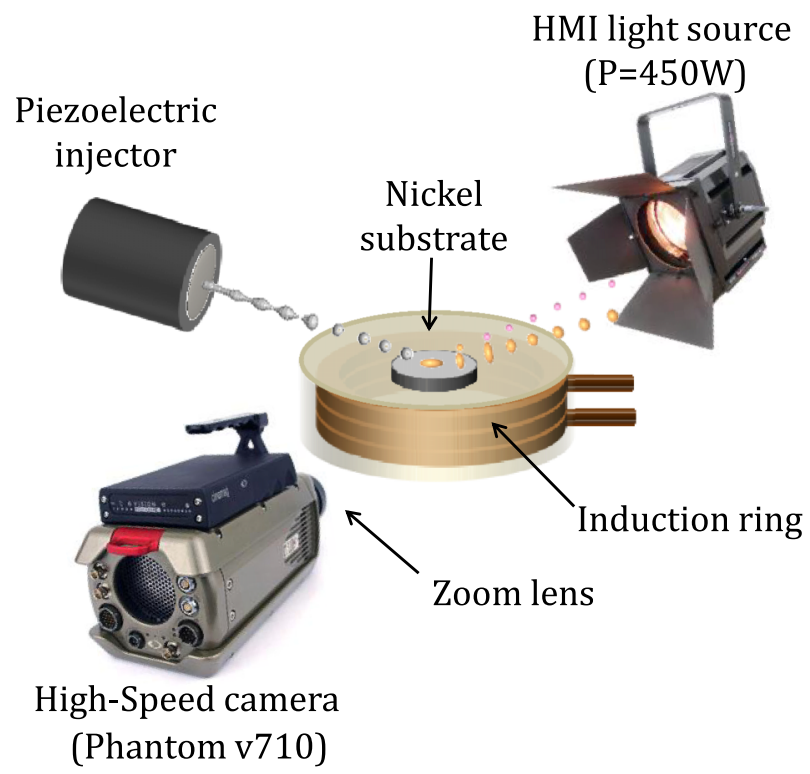

FIG. 1. Experimental setup used for the observation of the drop impact in the conditions of Leidenfrost boiling.

spaced droplets. The droplet generation results from the breakup of a cylindrical liquid jet through a Rayleigh-type instability. The vibrations of a piezoceramic attached to the injector body enable the instability of the liquid jet to be triggered which eventually results in the disintegration of the jet into a monodisperse droplet stream. In addition to the vibration frequency of the piezoceramic, the inlet pressure as well as the diameter of the injector orifice can be changed to adjust the drop size. The selected sizes for the orifice of the injector are $50 \mu \mathrm{m}$ and $100 \mu \mathrm{m}$. This enables the production of droplets ranging from $100 \mu \mathrm{m}$ to $300 \mu \mathrm{m}$ with a velocity set to a few $\mathrm{m} / \mathrm{s}$. High viscosity liquids like glycerol are relatively difficult to inject in a stable and periodical manner, and therefore, the volume fraction of glycerol in the water/glycerol mixtures was limited to $70 \%$. The droplet generator is rotated to modify the direction of the droplet stream and hence the component of the drop velocity normal to the wall. Impacts are not normal to the wall to avoid collisions between incoming and outgoing drops. Coalescence between consecutive drops can be sometimes observed at the wall if the spacing between them is small. The droplet temperature at the injection is set at $23^{\circ} \mathrm{C}$ for all the experiments reported in this study.

The wall is a $0.5 \mathrm{~mm}$ thick nickel plate heated by electromagnetic induction. Although the wall temperature was observed to have little influence on the drop dynamics in the film boiling regime, ${ }^{7,12}$ our experiments are performed well above the Leidenfrost temperature. The Leidenfrost temperature is known to increase with the impact velocity since the time for the formation of the vapor film becomes shorter. ${ }^{11}$ The wall temperature is fixed at $600{ }^{\circ} \mathrm{C}$, which is far beyond the dynamic Leidenfrost temperature for all the cases considered in the experiments. Surface roughness is also known to increase the Leidenfrost temperature and can alter droplet dynamics as the vapor film can be extremely thin at the initial stage of the impact. Karl and Frohn ${ }^{4}$ observed that the onset of the fragmentation is dependent on the surface roughness. The surface of the nickel sample is thus polished like a mirror. Profile measurements of the solid surface indicate a roughness average $R a$ of about $0.8 \mu \mathrm{m}$. It is the same roughness for each measurement as the sample is regularly polished between the experiments.

A high-speed camera (Phantom v710, Vision Research) is used to visualize the drop deformation. The droplets are illuminated from behind to get shadow images as illustrated in Fig. 2 . A zoom lens (OPTEM zoom 125C) enables us to observe the drop impacts with a high magnification. To visualize the complete deformation process, the resolution is about $5 \mu \mathrm{m}$ per pixel for $100 \mu \mathrm{m}$ drops. Given the small droplet size, the contact time is in the order of $100 \mu \mathrm{s}$. For this reason, time-resolved visualizations of the impacts require high acquisition rates close to 100000 
(a)

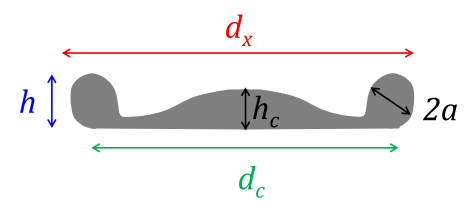

(b)

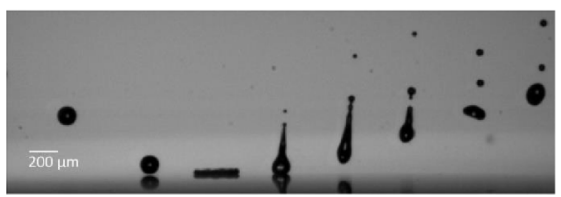

(c)

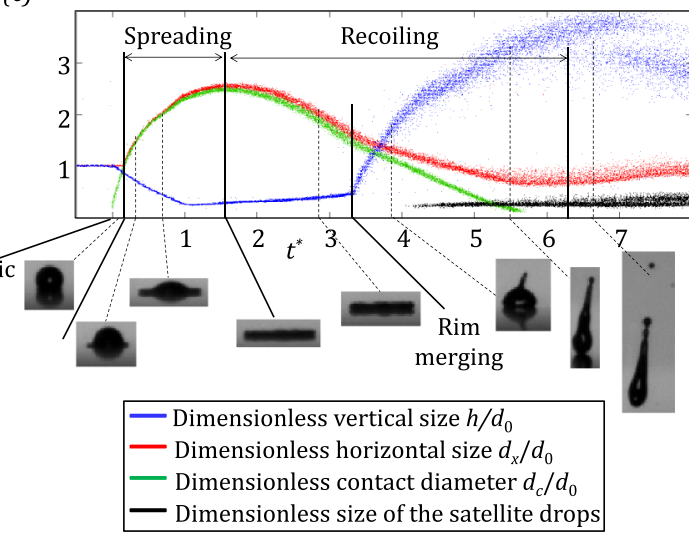

FIG. 2. Characterization of the deformation in the case of a drop made of $65 \%$ water and $35 \%$ glycerol by volume at $T_{0}=23^{\circ} \mathrm{C}\left(d_{0}=163 \mu \mathrm{m}, U_{0}=4.5 \mathrm{~m} / \mathrm{s}, W e=53\right.$, and $\left.R e=230\right)$. (a) Main parameters of the detection, (b) a typical shadow image, (c) reconstruction of the time evolution of the drop deformation from the shadow images.

fps. This is about 10 times faster than the frequency of the droplet injection; therefore, about 10 drops can be observed at the same time in an image (Fig. 2). A very bright light source (HMI $400 \mathrm{~W}$ ) is required so we can obtain contrasted shadow images at a short exposure time (presently, $1 \mu \mathrm{s}$ ) and thus avoid any motion blur. An image processing program is developed to characterize the most important parameters of the droplet deformation. The method used for the detection and the tracking of the drop trajectories has been described in more detail in Castanet et al. ${ }^{27}$ The extraction of the drop contours is adapted from the so-called Laplacian of the Gaussian (LoG) that is a common method for blob detection in the field of computer vision. Main adaptations are for the separation of overlapping particles and the size correction of defocused particles. Several characteristics of the particles are calculated after the detection including the equivalent diameter $d$ of a spherical particle that exhibits the same sectional area, the horizontal and vertical lengths of the particle (namely, the dimensions of its bounding box) which are denoted $d_{x}$ and $h$ (Fig. 2(a)). Also, the centers of the particles are localized. When the drop is in contact with the wall, the contact length $d_{c}$ is also computed. Here, the word "contact" is not well appropriate for the film boiling regime, since a small vapor layer separates the liquid and the solid wall. In this context, contact simply means that the distance between the liquid and the wall is below the resolution of the visualization system (typically, $5 \mu \mathrm{m} / \mathrm{pixel}$ ). In a second stage, the position of the particles is used to track their trajectories. The method also described in Castanet et al. ${ }^{27}$ is based on a Multiple Hypothesis Tracking (MHT) algorithm designed to track targets in cluttered environment. ${ }^{28}$ Tracks are formed upon the hypothesis that there is a certain regularity of the particle motion. For each drop (whose trajectory has been reconstructed), it is possible to plot the evolution of its characteristic dimensions $\left(h, d_{x}\right.$, and $\left.d_{c}\right)$ as a function of the time the droplet is in contact with the wall. Such a plot is presented in Fig. 2(c). Given the periodicity of the droplet streams, temporal evolutions are superimposed for every droplet. The curves in Fig. 2(c) correspond to the superimposition of about 200 drops. Each drop is seen about 20 times before leaving the field of view of the camera meaning that the data are the result of 1000 images.

\section{GENERAL PHENOMENA}

As shown in Fig. 2(c), the deformation process can be divided into three consecutive phases: kinematic, spreading, and recoiling. The kinematic phase is the initial stage of impact. A shock wave is created at impact because of liquid compression. ${ }^{29}$ During this phase, the spreading lamella is not yet visible. The bottom of the drop is stopped at impact, but the upper part of the drop is still moving with an undisturbed velocity so the drop looks like a cut sphere (Fig. 2(c)). The evolution of the drop shape can be easily described based on purely geometrical considerations. The 


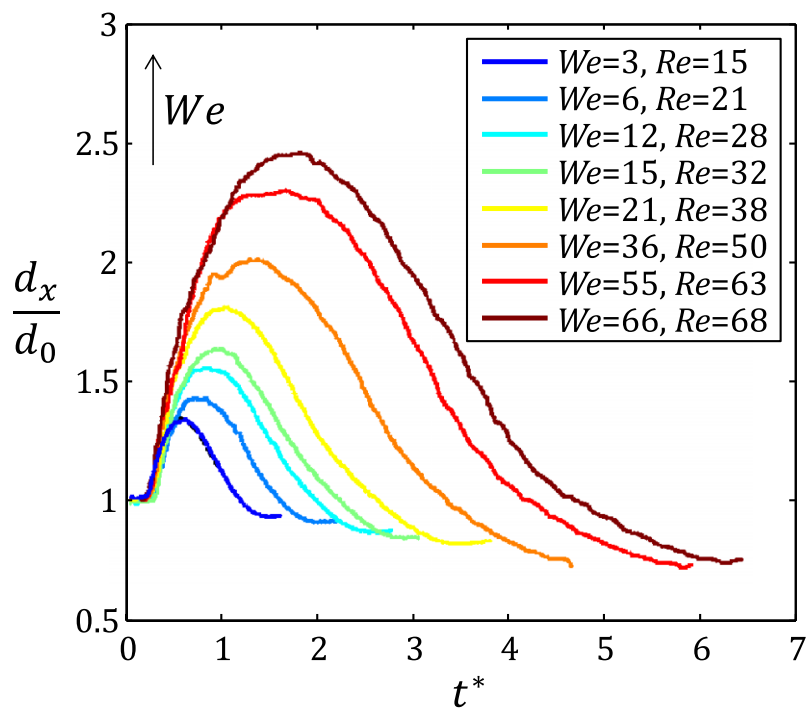

FIG. 3. Time evolution of the horizontal spreading $d_{x}$ for drops made of $40 \%$ water and $60 \%$ glycerol by volume at $T_{0}=23{ }^{\circ} \mathrm{C}$. The color lines are experimental data.

dimensionless height $h / d_{0}$ is given by $1-t^{*}$, where $t^{*}$ is the time made dimensionless by the initial droplet size $d_{0}$ and velocity $U_{0}$. The approximation of a truncated sphere yields the dimensionless contact diameter $d_{c} / d_{0}$ to be equal to $2 \sqrt{t^{*}}$, while the ratio $d_{x} / d_{0}$ remains equal to 1 . These evolutions are clearly visible in Figs. 3-5, where the geometrical parameters $d_{x} / d_{0}, d_{c} / d_{0}$, and $h / d_{0}$ are plotted as a function of the dimensionless time $t^{*}$ for different impact conditions. The drop evolution during the kinematic phase is universal; i.e., it does not depend on the drop size or the liquid properties. In Fig. 4, the dimensionless contact diameter $d_{c} / d_{0}$ is displayed on a semi-log scale, which reveals that the scaling progressively shifts from $t^{* 0.5}$ to a higher exponent. The kinematic phase is limited to $t^{*}<0.1$, which is in line with previous observations. ${ }^{30}$

In the spreading phase, a lamella is ejected from the base of the drop and forms a thin film with a bounding rim because of the capillary effects (Fig. 2(c)). The drop evolution in this phase is a

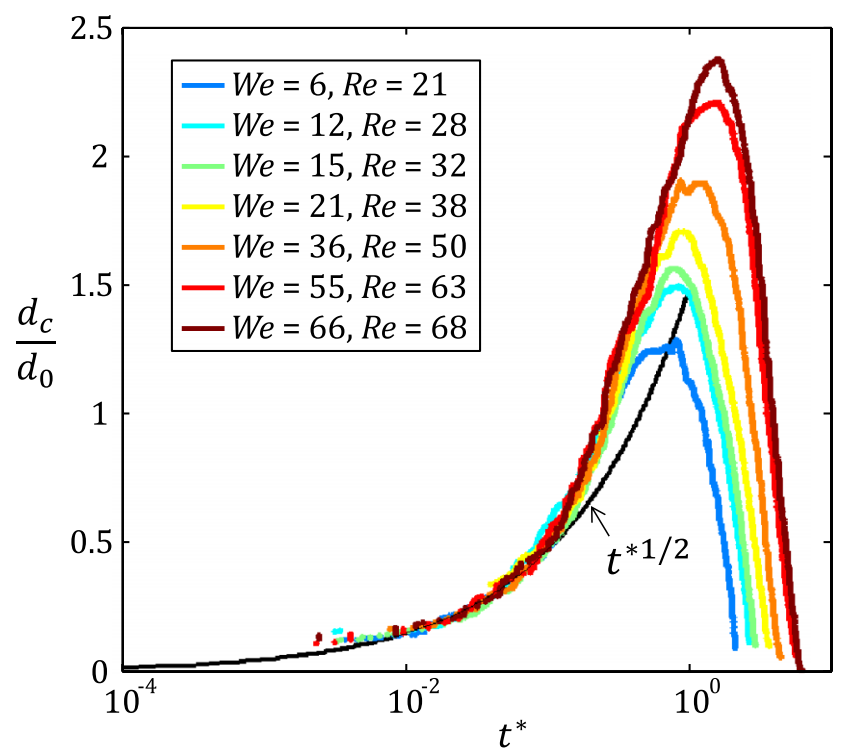

FIG. 4. Time evolution of the contact diameter $d_{c}$ for drops made of $40 \%$ water and $60 \%$ glycerol by volume at $T_{0}=23^{\circ} \mathrm{C}$. The color lines: experimental data; the black line: illustration of an evolution as $t^{* 1 / 2}$. 

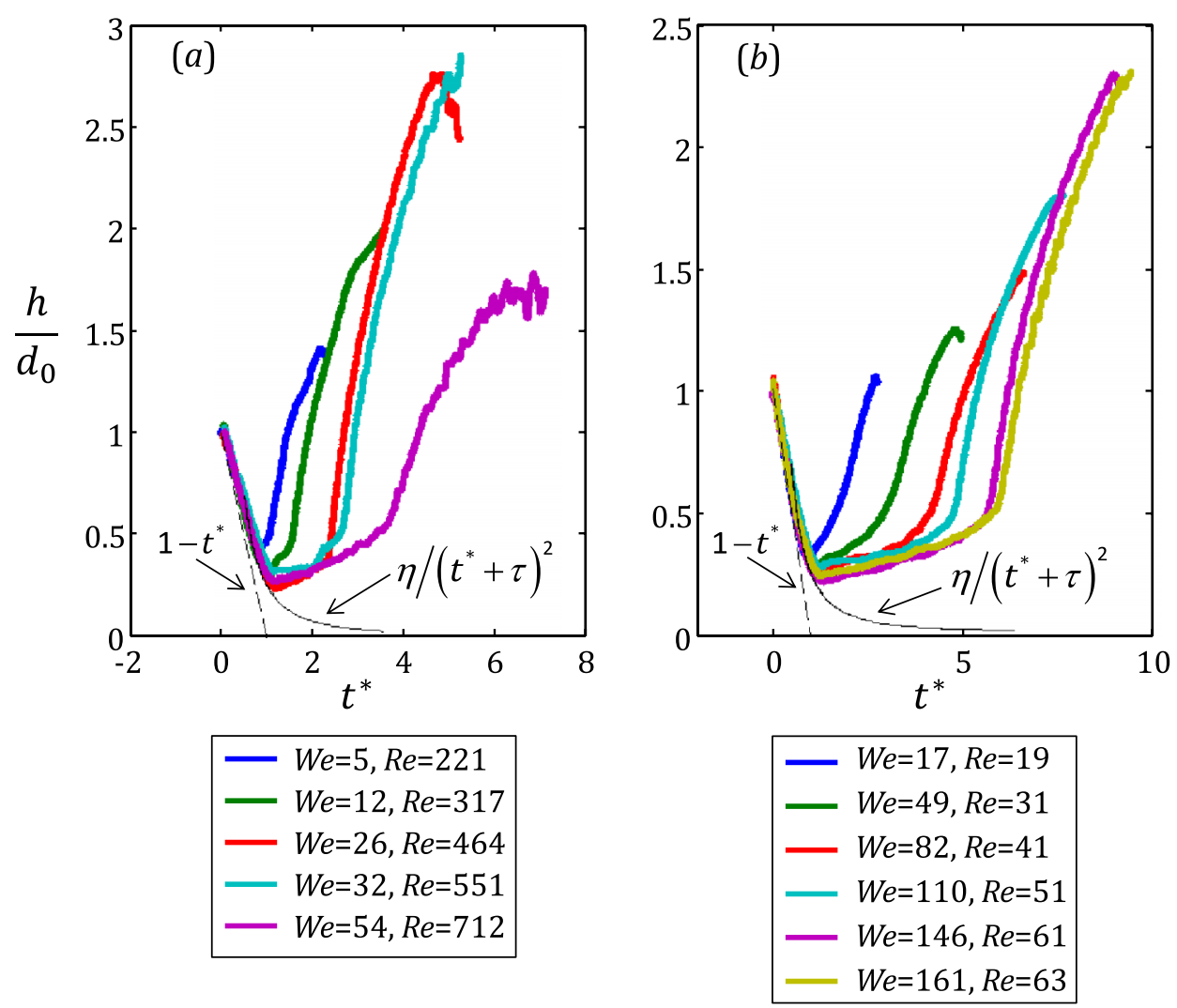

FIG. 5. Time evolution of the droplet height $h$ for different liquids: (a) water, (b) mixture of $70 \%$ glycerol $/ 30 \%$ water by volume at $T_{0}=23^{\circ} \mathrm{C}$. The color lines: experimental data; the dotted line: $1-t^{*}$, theoretical evolution in the kinematic phase; the solid black line: droplet thickness based on Eq. (10) at $r=0$ with $\eta=0.39$ and $\tau=0.25$.

function of $W e$ as illustrated in Figs. 3-5. The maximum spreading diameter is smaller and reached earlier for the low Weber numbers (Fig. 3).

In Fig. 5, the lamella thickness $h$ at the axis $r=0$ is found to behave as $1 / t^{* 2}$. However, after a certain time, the top of the lamella becomes hidden by the rim because the images are only side views. A clear change in the slope of the blue curves can be observed in Fig. 2(c) when the top of the lamella becomes hidden at $t^{*}=1.1$ and when it reappears at $t^{*}=3.4$. Measurements reveal that the rim thickness is increasing at an almost constant rate. The height of the rim appears to decrease with the Weber number. However, this does not mean that the rim is less voluminous as the spreading radius $d_{x}$ increases with $W e$ (Fig. 3). After the spreading phase, the drop begins to recede radially. The recoil is initiated by the dominating surface forces which restore the drop shape minimizing the free surface energy. During this phase, the rim merging develops with the formation of a vertical finger-like jet. If $W e$ is high enough, one or two small drops may detach at the top of this liquid jet, as visible in Fig. 2(c). The reproducibility of this fragmentation process is not high. The time for the formation of satellite drops may fluctuate during experiments. Also, the scattering of the data was found to be more pronounced in the recoiling phase. Surface oscillations are gradually damped after the drop leaves the wall.

\section{MODELING OF THE DROP SPREADING}

The model for the drop spreading is adapted from an approach developed by Roisman et al. to describe the spreading of drops on dry and wetting surfaces ${ }^{24,25}$ and the binary collisions of drops ${ }^{26}$ given the similarities between these different processes. It should be noted that the definition for the Weber and Reynolds numbers is generally different in the head-on collisions of equal drops. The 
Weber and Reynolds numbers used to describe the binary collision of drops are usually based on the relative velocity between the drops, which is the double of the "impact" velocity onto the symmetry plane.

\section{A. Description of the flow in the lamella}

The axisymmetric spreading of a thin free liquid sheet is used as the basis for the description of the lamella. The flow in the sheet and the evolution of the sheet thickness can be determined by considering the mass and momentum balances in the cylindrical coordinate system $(r, \phi, z)$ fixed at the axis of the sheet. ${ }^{21}$ In the following, the thickness of the sheet is denoted by $h(r, t)$ while $u_{r}(r, t)$ refers to the radial velocity averaged through the sheet cross section. The mass balance expresses the change in volume of a sheet element due to the mass volume flux gradient in the radial direction,

$$
\frac{\partial(r h)}{\partial t}+\frac{\partial\left(r h u_{r}\right)}{\partial r}=0
$$

The momentum balance accounts for the capillary pressure applied to the free surface of the sheet and the internal stresses applied to the cross section of the sheet element,

$$
\rho \frac{\partial\left(r h u_{r}\right)}{\partial t}+\rho \frac{\partial\left(r h u_{r}^{2}\right)}{\partial r}=\frac{\partial\left(r h \sigma_{r r}\right)}{\partial r}-h \sigma_{\phi \phi}+p_{\sigma} r \frac{\partial h}{\partial r} .
$$

In this expression, $\sigma_{r r}$ and $\sigma_{\phi \phi}$ are the components of the stress tensor in the radial and polar directions, respectively, and $p_{\sigma}$ is the pressure jump which appears at the surface of the liquid sheet due to surface tension. Given the small thickness of the liquid sheet, a good way of approximately defining the axial component of the stress tensor is to consider that $\sigma_{z z}=-p_{\sigma}$ throughout a cross section of the sheet. Assuming the liquid incompressibility $\left(\sigma_{r r}+\sigma_{\phi \phi}+\sigma_{z z}=-3 p\right)$, the stress components $\sigma_{r r}=-p+2 \mu \partial u_{r} / \partial r$ and $\sigma_{\phi \phi}=-p+2 \mu u_{r} / r$ can be determined by

$$
\begin{gathered}
\sigma_{r r}=-p_{\sigma}+2 \mu\left(\frac{u_{r}}{r}+2 \frac{\partial u_{r}}{\partial r}\right), \\
\sigma_{\phi \phi}=-p_{\sigma}+2 \mu\left(2 \frac{u_{r}}{r}+\frac{\partial u_{r}}{\partial r}\right) .
\end{gathered}
$$

Using the expressions of $\sigma_{r r}, \sigma_{\phi \phi}$, and mass conservation (1), momentum equation (2) can be rewritten in the form

$$
\rho\left(\frac{\partial u_{r}}{\partial t}+u_{r} \frac{\partial u_{r}}{\partial r}\right)=-\frac{\partial p_{\sigma}}{\partial r}+4 \mu\left\{\frac{1}{h} \frac{\partial h}{\partial r}\left[\frac{u_{r}}{2 r}+\frac{\partial u_{r}}{\partial r}\right]+\frac{\partial}{\partial r}\left[\frac{1}{r} \frac{\partial\left(r u_{r}\right)}{\partial r}\right]\right\} .
$$

For high Reynolds and Weber numbers, the spreading liquid sheet rapidly becomes thin and smooth. The capillary pressure $p_{\sigma}$ can be neglected in Eq. (5) and bearing in mind the fact that the gradient of the sheet thickness is small $\left(h^{-1} \partial h / \partial r \ll 1\right)$, further simplifications can be made in Eq. (5),

$$
\frac{\partial u_{r}}{\partial t}+u_{r} \frac{\partial u_{r}}{\partial r}=4 v \frac{\partial}{\partial r}\left[\frac{1}{r} \frac{\partial\left(r u_{r}\right)}{\partial r}\right] .
$$

This equation still takes into account some effects of the viscosity which have not been totally eliminated. It is similar to the Navier-Stokes equation in the radial direction with $4 v$ being the effective kinematic viscosity in the sheet. Equation (6) can be resolved by applying self-similar solutions as follows:

$$
u_{r}=\frac{f(\xi) r}{t+\tau_{0}},
$$

with $\xi=r / \sqrt{v\left(t+\tau_{0}\right)}$, and $\tau_{0}$ is a constant related to the velocity gradient at the initial time. An ordinary differential equation satisfied by $f(\xi)$ is obtained by substituting Eq. (7) in Eq. (6). The general solution of this non-linear ODE is given by Eq. (4.10) in Roisman et al. ${ }^{26}$ As $\xi$ approaches 0 
(i.e., for large times and small radii), the function $f(\xi)$ tends toward the integration constant $A$ and the radial velocity in the sheet can be expressed as the asymptotic solution,

$$
u_{r}=\frac{A r}{t+\tau_{0}} \quad \text { with } \quad \xi \rightarrow 0 .
$$

In this expression, fixing $A$ equals 1 allows to find a velocity field identical to the inviscid asymptotic solution formerly derived by Yarin and Weiss. ${ }^{20}$ It should be noted that the case $A=1$, in addition to correspond to the inviscid asymptotic solution, still satisfies Navier-Stokes equation (5) with its viscous term. In principle, values of $A$ slightly different from 1 are also possible. However, as pointed out by Roisman et al. ${ }^{26}$ these would lead to solutions where $f(\xi)$ rapidly diverges from unity as $\xi$ increases.

The distribution of the liquid film thickness $h(r, t)$ can be specified by substituting the asymptotic solution for radial velocity (8) in mass balance (1). A general solution of the partial differential equation verified by $h(r, t)$ has the following form:

$$
h=\frac{\tau_{0}^{2 A}}{\left(t+\tau_{0}\right)^{2 A}} H_{0}\left[\frac{r \tau_{0}^{A}}{\left(t+\tau_{0}\right)^{A}}\right],
$$

where $H_{0}$ is an universal function that does not depend a priori on the Reynolds and Weber numbers. Equation (9) predicts that the liquid thickness at $r=0$ evolves as $1 / t^{2 A}$. As shown in Fig. 5, there is experimental evidence that the lamella thickness at $r=0$ behaves as $1 / t^{2}$. Consequently, a value of $A=1$, corresponding to the inviscid asymptotic solution, is expected to effectively describe the radial velocity inside the droplets and the evolution of the lamella thickness. In this respect, the spreading of droplets in the Leidenfrost boiling is not so different from the impact on a wetting surface or the binary collision of droplets, for which the lamella thickness has also been reported to behave as $1 / t^{2} .{ }^{21,31,32}$ Based on numerical simulations, Roisman et al., ${ }^{21}$ proposed to approximate the thickness distribution of the lamella using a Gaussian profile,

$$
h^{*}=\frac{h}{d_{0}}=\frac{\eta}{\left(t^{*}+\tau\right)^{2}} \exp \left(-\frac{6 \eta r^{* 2}}{\left(t^{*}+\tau\right)^{2}}\right)
$$

where $\eta=0.39, \tau=0.25, r^{*}=r / d_{0}$, and $t^{*}=t U_{0} / d_{0}$. Eggers et al. ${ }^{22}$ also evaluated $H_{0}$ using numerical simulations. Their result compares very well with (10), although they did not use a Gaussian function to approximate $H_{0} \cdot{ }^{31}$ According to Eq. (10), the shape of the lamella does not depend on Reynolds and Weber numbers which is supported theoretically at high Reynolds and Weber numbers. As the flow is mainly governed by inertia, it is understandable that the dimensionless shape of the lamella should not depend on the impact conditions. The numerical results reported by Roisman et al. ${ }^{21}$ suggested that approximation (10) is acceptable for $R e>25$. Also, they carried out this verification for drop impacting on a symmetry plane and for $W e>100$ and $R e>10$ whereas the Reynolds and Weber numbers are sometimes lower in the work presented in this paper (Table I). Nevertheless, good agreements with Eq. (10) can be observed in Fig. 5 at the central axis $\left(r^{*}=0\right)$ beyond $t^{*}=0.5$. However, this validation is limited to the central region of the lamella and to the time period when the top of the lamella is above the rim. Near the edge of the lamella, asymptotic solution (8) and thickness profile (10) can be less reliable since $\xi$ takes more time to approach 0. Basically, Eqs. (8) and (10) should be considered as approximations which remain valid for high Reynolds and Weber numbers in the limit as $\xi \rightarrow 0$. Recently, Lastakowski et al..$^{33}$ measured the thickness profile of the lamella based light absorption technique. Since the droplets are observed from the top in their method, the thickness profile can be observed over a large time period compared with side-view imaging techniques. Based on the experiments, Lastakowski et al. ${ }^{33}$ found that $h^{*}\left(r^{*}=0\right) \approx 0.4 /\left(t^{*}+0.5\right)^{2}$ shows good agreement with their measurements of the central thickness of the lamella. However, this fitted expression was established for relatively large times $t^{*}>1.5$ and yields a central thickness significantly smaller than in our measurements. 
TABLE I. Overview of the experimental conditions under investigation.

\begin{tabular}{lcccrr}
\hline \hline & $d_{0}(\mu \mathrm{m})$ & $U_{0}(\mathrm{~m} / \mathrm{s})$ & $W e$ & $R e$ & $O h$ \\
\hline Water & $101-163$ & $1.57-6.10$ & $4-52$ & $175-810$ & $9.85 \times 10^{-3}$ \\
Ethanol & $109-139$ & $0.84-3.10$ & $3-40$ & $71-256$ & $2.5 \times 10^{-2}$ \\
Water 65\%-glycerol 35\% & $160-164$ & $1.3-5$ & $4-66$ & $65-225$ & $3.16 \times 10^{-2}$ \\
Water 56\%-glycerol 44\% & $145-154$ & $1-4.5$ & $3-50$ & $34-140$ & $5.02 \times 10^{-2}$ \\
Water 50\%-glycerol 50\% & $114-129$ & $2-6.7$ & $9-88$ & $38-111$ & $8.22 \times 10^{-2}$ \\
Water 40\%-glycerol 60\% & $170-200$ & $1-5$ & $3-86$ & $15-78$ & $1.19 \times 10^{-1}$ \\
Water 35\%-glycerol 65\% & $98-159$ & $3-7.7$ & $15-152$ & $17-67$ & $1.93 \times 10^{-1}$ \\
Water 30\%-glycerol 70\% & $230-297$ & $1.8-5.5$ & $14-160$ & $17-63$ & $2.16 \times 10^{-1}$ \\
\hline \hline
\end{tabular}

\section{B. Propagation of the rim}

The dynamics of the rim is inferred from the mass balance of the rim and the momentum equation applied to the rim..$^{25,26}$ The momentum balance accounts for the capillary force $F_{\sigma}$ at the free surfaces, the inertia of the liquid entering the $\operatorname{rim} F_{i l r}$, and the viscous stress $F_{v}$ at the interface between the lamella and the rim (i.e., between the light grey and dark grey regions in Fig. 6),

$$
\rho W_{R} \ddot{R}_{R}=F_{i l r}-F_{v}-F_{\sigma} \text { at } r=R_{R},
$$

where $R_{R}$ and $W_{R}$ are, respectively, the rim radius and the rim volume. The inertia of the liquid entering the control volume in dark grey corresponding to the rim in Fig. 6 can be expressed as

$$
F_{i l r}=\rho\left(\dot{R}_{R}-u_{\text {rim }}\right)^{2} S_{l R},
$$

where $u_{\text {rim }}$ corresponds to the averaged radial velocity at $r=R_{R}$ given by Eq. (8) and $S_{l R}=$ $2 \pi R_{R} h\left(R_{R}\right)$ is the separation surface between the lamella and the rim. The capillary force $F_{\sigma}$ can be determined by

$$
F_{\sigma}=S_{l R} \sigma\left(\frac{2}{h}\right)=4 \pi R_{R} \sigma .
$$

Neglecting $F_{v}$ and $\ddot{R}_{R}$ in Eq. (11), the balance between the forces of inertia and surface tension leads to $\rho h\left(\dot{R}_{R}-u_{r}\right)^{2}=2 \sigma$, which is a reminiscent of the Culick's law for the receding velocity of a liquid film. ${ }^{34}$

The force $F_{v}$ in Eq. (11) originates from the viscous stresses at the separation surface between the lamella and the rim. Obviously, viscous dissipation is predominant within the rim, but the viscous forces arising from the liquid motion inside the rim do not affect the motion of the rim, as they correspond to internal forces. Also, in the model, it is considered that no force is exerted by the vapor film directly on the rim surface. The vapor film exclusively acts upon the lamella because of the large contact area between both of them (much larger than the contact area with the rim). In the following, the lamella is first assumed to slide perfectly over the vapor film. This assumption considerably simplifies the problem, since no boundary layer would develop at the bottom edge of the spreading lamella, meaning that radial velocity (8) and thickness profile (10) can be applied without any restriction other than those already mentioned in Sec. IV A (high $R e$ and $W e$ ). If

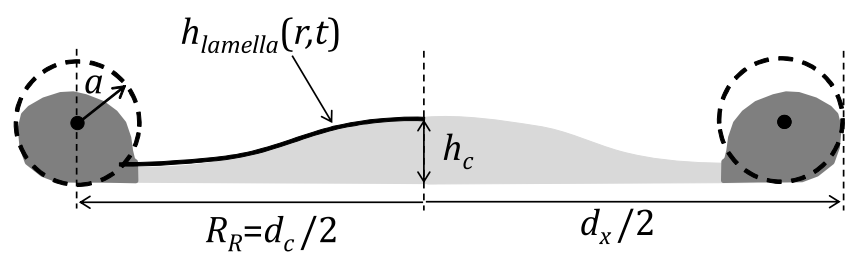

FIG. 6. Schematic view of the droplet geometry considered in the modeling: a torus of circular section (dotted lines) featuring the rim (dark grey region) bounded by a Gaussian-shape lamella (light grey region). 
frictions are exerted on the lower side of the droplet, the shear stress over the lamella will produce a boundary layer in the lamella which needs to be taken into account by modifying the inertia of the liquid entering the rim. This scenario will be further discussed in Secs. VI and VII. Substituting radial velocity (8) with $A=1$ in Eq. (3), the stress tensor component $\sigma_{r r}$ becomes

$$
\sigma_{r r}=6 \mu /\left(t+\tau_{0}\right) .
$$

In this expression, the capillary pressure $p_{\sigma}$ is neglected as it is very small for the spreading of a thin lamella at high Weber number. From Eq. (14), the viscous force $F_{v}$ can be expressed as

$$
F_{v}=S_{l R} \sigma_{r r} .
$$

It is interesting to note that viscous stresses are not totally eliminated even though the inviscid flow solution $(A=1)$ is used in the model and no friction is acting on the lower side of the droplet (assumption of perfect sliding over the vapor layer).

Momentum equation parameters (11) are made dimensionless using the droplet diameter $d_{0}$ and the impact velocity $U_{0}$. This yields the following expression:

$$
\frac{W_{R}^{*}}{2 \pi R_{R}^{*} h_{L}^{*}} \ddot{R}_{R}^{*}=\left[u_{r i m}^{*}-\dot{R}_{R}^{*}\right]^{2}-\frac{6}{\operatorname{Re}\left(t^{*}+\tau\right)}-\frac{2}{W e h_{L}^{*}},
$$

with $h_{L}^{*}=h^{*}\left(R_{R}^{*}\right)$ and $u_{\text {rim }}^{*}=R_{R}^{*} /\left(t^{*}+\tau\right)$. Momentum equation (16) is supplemented by the conservation of the liquid volume. The non-dimensional volume of the rim $W_{R}^{*}$ is determined from lamella thickness (10),

$$
W_{R}^{*}=\frac{\pi}{6}-\int_{0}^{R_{R}^{*}} 2 \pi h^{*} d r=\frac{\rho}{6} \exp \left[-\frac{6 \eta R_{R}^{* 2}}{\left(t^{*}+\tau\right)^{2}}\right] .
$$

Equations (16) and (17) form a set of equations which need to be solved to describe the dynamics of the drop spreading. A parallel can be drawn with the head-on collision of identical drops, for which there is also a perfect sliding condition at the symmetry plane where the two drops enter in collision. In Roisman et al., ${ }^{26}$ equations describing the head-on collisions are basically the same except that the capillary term in Eq. (16) is replaced by $1 /\left(W e h_{L}^{*}\right)$, and $W_{R}^{*}$ is multiplied by two. Eggers et al. ${ }^{22}$ and Lastakowski et al. ${ }^{33}$ derived an equation similar to Eq. (16) to describe the rim propagation, but they neglect the viscous force $F_{v}$ in their analyses. In Eggers et al. ${ }^{22}$ the inertia of the liquid entering the rim is also corrected for the boundary layer that develops in the lamella. In this study dealing with drop impacts on hydrophobic substrates, frictions appear due to the no-slip condition at the wall on which the droplet is deposited.

Ordinary differential equation (16) is solved using the Runge-Kutta method. For the resolution, the initial conditions for $R_{R}^{*}$ and $\dot{R}_{R}^{*}$ are fixed at $t_{i}^{*}=0.5$ since thickness profile (10) is relevant at that time, at least close to the central axis (Fig. 5). Generally, setting the initial conditions at $t^{*}=0.5$ can be a problem. But, in our case, the initial conditions can be obtained from the experimental profiles of the spreading diameter $d_{x}$. The initial values of $R_{R}$ and $\dot{R}_{R}$ are adjusted so that $d_{x}$ has the same value and rate of expansion in the simulations and in the measurements at $t^{*}=0.5$. Finally, assuming that the rim is a torus with a circular section of radius $a$ (Fig. 6),

$$
W_{R}=\left(2 \pi R_{R}\right) \pi a^{2} .
$$

The spreading diameter $d_{x}$ is obtained by

$$
d_{x}=2\left(R_{R}+a\right) .
$$

Simulations are stopped when $R_{R}=a$ which corresponds to the merging of the inner edge of the rim in the receding phase.

\section{MAXIMUM SPREADING DIAMETER: SCALING ANALYSIS}

The liquids used in the experiments are water, ethanol, and several mixtures made of water and glycerol. The main properties of these liquids are summarized in Table II. Two temperatures 
TABLE II. Physical properties of the liquids studied. For the water/glycerol solutions, proportions of the mixtures are indicated in volume fractions and the physical properties are extrapolated from "Physical properties of glycerine and its solutions." 35

\begin{tabular}{|c|c|c|c|c|c|c|}
\hline & \multicolumn{3}{|c|}{$23^{\circ} \mathrm{C}$} & \multicolumn{3}{|c|}{$100^{\circ} \mathrm{C}$} \\
\hline & $\rho\left(\mathrm{kg} \cdot \mathrm{m}^{-3}\right)$ & $\mu(\mathrm{mPa} \cdot \mathrm{s})$ & $\sigma\left(\mathrm{mN} \cdot \mathrm{m}^{-1}\right)$ & $\rho\left(\mathrm{kg} \cdot \mathrm{m}^{-3}\right)$ & $\mu(\mathrm{mPa} \cdot \mathrm{s})$ & $\sigma\left(\mathrm{mN} \cdot \mathrm{m}^{-1}\right)$ \\
\hline Water & 997.6 & 0.94 & 72.3 & 958 & 0.28 & 60.5 \\
\hline Ethanol & 789.7 & 1.14 & 22.0 & $738.8^{\mathrm{a}}$ & $0.41^{\mathrm{a}}$ & $17.3^{\mathrm{a}}$ \\
\hline Glycerol & 1259.8 & 924.00 & 63.1 & 1209.3 & 14.8 & 58.6 \\
\hline Water $65 \%$-glycerol $35 \%$ & 1099.1 & 3.51 & 69.2 & 1053.3 & 0.7 & 60.1 \\
\hline Water $56 \%$-glycerol $44 \%$ & 1124.1 & 5.42 & 68.4 & 1076.8 & 0.91 & 60.0 \\
\hline Water $50 \%$-glycerol $50 \%$ & 1140.5 & 7.92 & 67.9 & 1092.7 & 1.12 & 60 \\
\hline Water $40 \%$-glycerol $60 \%$ & 1166.9 & 14.05 & 66.9 & 1118.1 & 1.56 & 59.7 \\
\hline Water $35 \%$-glycerol $65 \%$ & 1179.6 & 20.16 & 66.4 & 1130.4 & 1.94 & 59.5 \\
\hline Water $30 \%$-glycerol $70 \%$ & 1192.0 & 30.39 & 65.9 & 1142.2 & 2.39 & 59.3 \\
\hline
\end{tabular}

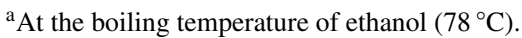

are considered in this table: the injection temperature $T_{0}=23^{\circ} \mathrm{C}$ and the saturation temperature $T_{\text {sat }}$. The properties of pure glycerol are also added for comparison. Ethanol has a viscosity close to that of water, but a much lower surface tension. For glycerol/water mixtures, the surface tension is moderately affected by the composition while the viscosity is heavily dependent on the mixture fraction. Glycerol and water do not have the same volatility which is a source of difficulty regarding to evaluate conditions at the drop surface (temperature and composition). Given the short duration of the drop/wall interaction, the variations of the bulk properties $(\rho, \mu)$ can be assumed to be limited. This idea is backed up by recent measurements which show that drop heating is limited to a few ${ }^{\circ} \mathrm{C}$ during a rebound. ${ }^{36-38}$ In the following, liquid properties are taken at the injection temperature $T_{0}=23^{\circ} \mathrm{C}$ to calculate $W e$ and $R e$. The experimental conditions are summarized in Table I and cover the rebound regime including partial rebounds (Fig. 2).

Figure 7 displays the maximum spreading factor $\beta_{\max }=d_{x, \max } / d_{0}$ as a function of the Weber number for the different investigated liquids. A clear dependence on $W e$ can be noted for all the liquids including the most viscous mixtures of water and glycerol, which indicates that kinetic energy is not completely dissipated during the spreading phase. The spreading factor is found to be larger for the less viscous liquids. Further analysis reveals that $\beta_{\max }$ varies roughly as $R e^{1 / 5}$ for

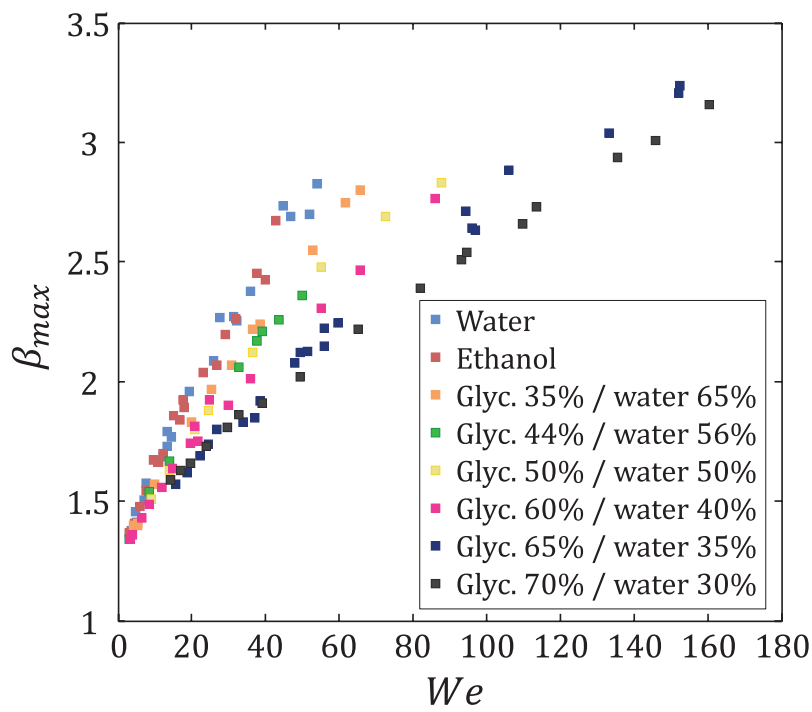

FIG. 7. Dependence of the maximum spreading factor $\beta_{\max }=d_{x, \max } / d_{0}$ on the Weber number. Points: experimental data. 


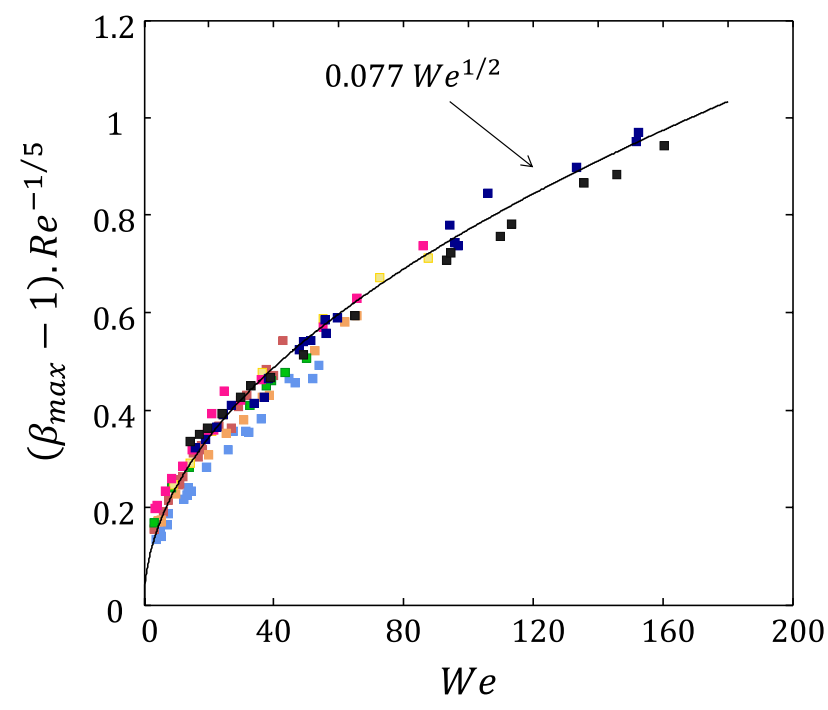

\begin{tabular}{|ll|}
\hline - Water & Glyc. $50 \%$ / water $50 \%$ \\
- Ethanol & - Glyc. $60 \%$ / water $40 \%$ \\
" Glyc. $35 \%$ / water $65 \%$ & - Glyc. $65 \%$ / water $35 \%$ \\
- Glyc. $44 \%$ / water $56 \%$ & - Glyc. $70 \%$ / water $30 \%$ \\
\hline
\end{tabular}

FIG. 8. Variation of $\left(\beta_{\max }-1\right) / R e^{1 / 5}$ as a function of $W e$. Squares: experimental data; the solid line: best fitting with $W e^{1 / 2}$.

the high viscous liquids. This trend is illustrated in Fig. 8 where the parameter $\left(\beta_{\max }-1\right) / R e^{1 / 5}$ is plotted as a function of $W e$. Measurement points are almost superimposed for all the liquids with the exception of water and ethanol. For the high viscous liquids (water/glycerol mixtures), the spreading factor can be determined by $\beta_{\max } \approx 1+7.7 \cdot 10^{-2} \mathrm{Re}^{1 / 5} \mathrm{~W} e^{1 / 2}$. For water and ethanol, the Reynolds number seems to have a more limited influence on spreading. Data from Tran et al. ${ }^{7}$ and Chaves et al. ${ }^{39}$ are added in Fig. 9 to extend the range of Reynolds numbers. These correspond to millimetric drops with a Reynolds number about 4 times higher than that in the experiments described in this paper. The maximum spreading factor $\beta_{\max }$ appears to correlate with $W e^{1 / 2}$ in Fig. 9. This scaling is different from $W e^{0.39}$ which was proposed by Tran et al. ${ }^{7}$ based on the same data. The difference is due to the subtraction of 1 to $\beta_{\max }$ in the plot of Fig. 9. This subtraction is justified by the fact that $\beta_{\max }$ tends to 1 at small $W e$.

A scaling of $\beta_{\max }$ as $W e^{1 / 2}$ is classically interpreted as being the result of a pure transfer of kinetic energy (of the order of $\rho d_{0}^{3} U_{0}^{2}$ ) into surface energy. Neglecting the surface area of the drop edge, the energy conservation is $\rho d_{0}^{3} U_{0}^{2} \sim \sigma d_{x, \max }^{2}$ which yields $\beta_{\max } \sim W e^{1 / 2}$. This application of the principle of conservation of mechanical energy assumes a zero velocity of the flow at the time of the maximum spreading. However, the flow velocity never totally vanishes inside the droplet. The maximum spreading diameter is reached when $\dot{R}_{R} \approx 0$. During the spreading phase, the velocity of the rim $\dot{R}_{R}$ is smaller than the velocity of the liquid in the lamella, which then leads to the rim growth. The velocity in the lamella decreases as $1 / t$, and hence, the inertia of the liquid entering the rim becomes eventually unable to counteract the capillary force and the friction. This eventually causes the rim to recede in accordance with Eq. (16) of the mechanistic model.

The power $1 / 5$ of the Reynolds number is known to scale the spreading factor of viscous droplets which impact on wetting surfaces. In the limit of liquids of high viscosity, the kinetic energy of the drop is completely dissipated during the spreading phase. The dissipation of energy scales as $\mu\left(U_{0} / h_{d}\right) d_{x, \max }^{3}, h_{d}$ being the thickness of the drop at the maximum spreading. Together with volume conservation $\left(h_{d} d_{x, \max }^{2} \sim d_{0}^{3}\right)$, this yields $d_{x, \max } \sim d_{0} R e^{1 / 5} \cdot{ }^{17,29}$ However, the scaling argument does not hold here as $R e^{1 / 5}$ is multiplied by $W e^{1 / 2}$ in the correlation obtained for $\beta_{\max }$. 


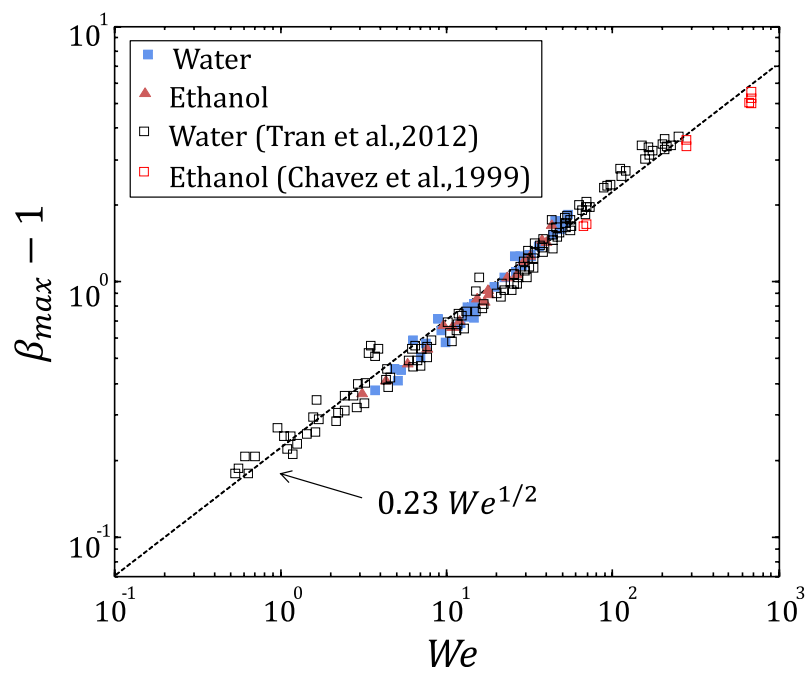

FIG. 9. Dependence of $\beta_{\max }-1$ on the Weber number for water and ethanol droplets. Filled symbols: data from experiments concerning droplets with a diameter of about $100 \mu \mathrm{m}$; hollow symbols: data from experimental studies of droplets sizing a few millimeters; the dotted line: best fitting with $W e^{1 / 2}$.

\section{APPLICATION OF THE THEORETICAL MODEL}

The model of the rim dynamics presented in Sec. IV is compared to the experimental deformations. Figure 10 gives the results for several impacts corresponding to water, and two mixtures made of water and glycerol. In this figure, the solid lines correspond to experimental data while
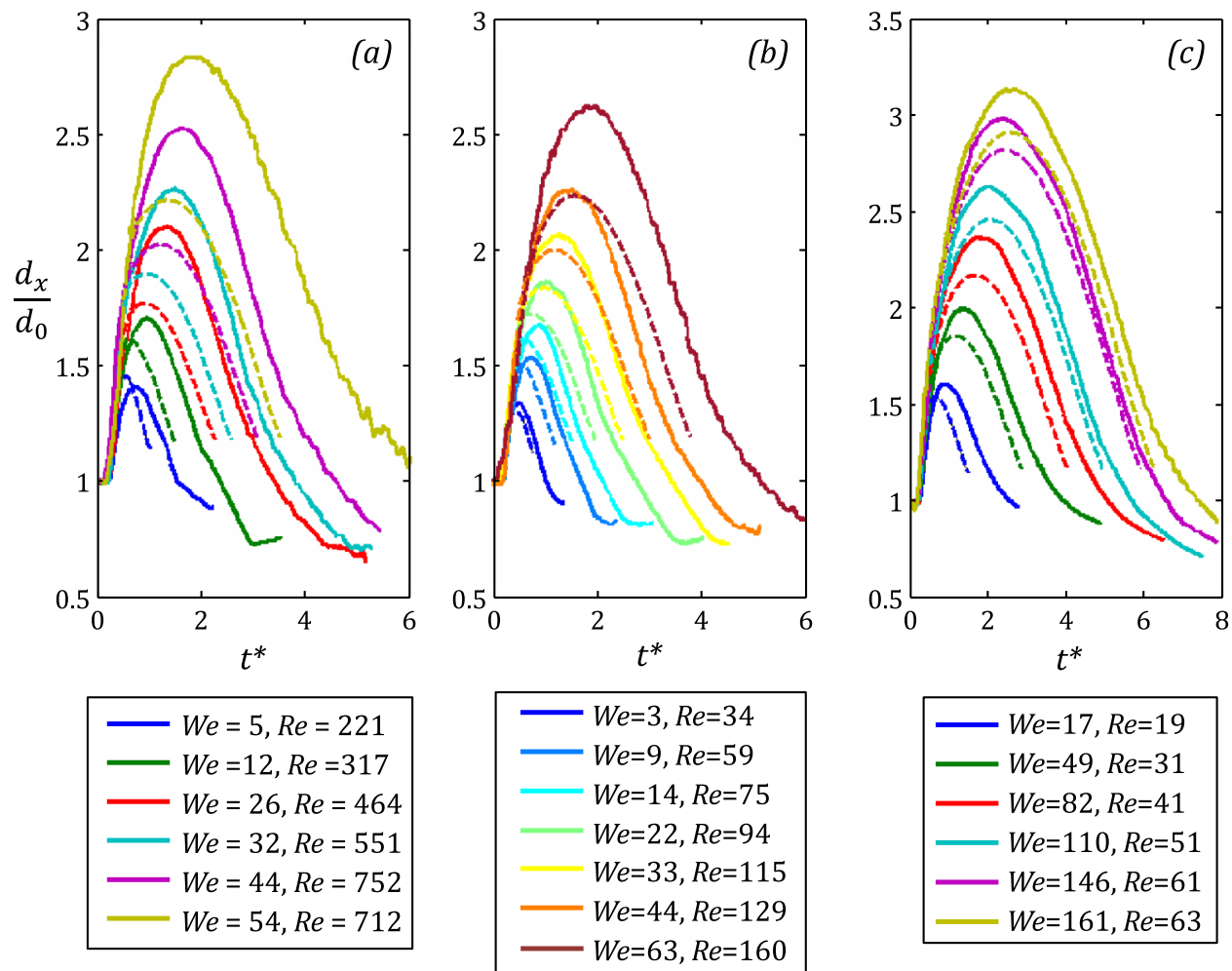

FIG. 10. Time evolution of the spreading factor $\beta=d_{x} / d_{0}$ for several Weber numbers and three liquids: (a) water, (b) mixture of $44 \%$ glycerol $/ 56 \%$ water, (c) mixture of $70 \%$ glycerol $/ 30 \%$ water. Solid lines: experimental data; dotted lines: solutions of Eq. (16) assuming the inviscid flow solution (Eq. (8) with $A=1$ ) to calculate the velocity of the liquid entering the rim. 
the dotted lines represent our simulations. The theoretical model with $A=1$ in Eq. (8) appears to systematically underestimate the amplitude of the deformation and the difference with experimental results is greatest for water, the least viscous liquid. This result seems rather counterintuitive. If not comparable to the measurements, the deformation was expected to overestimate the experimental spreading especially for the more viscous liquids assuming inviscid flow solution (8) and a perfect sliding over the vapor layer. The influence of the temperature on the liquid properties $(\rho, \mu, \sigma)$ is also tested to determine whether the heat transfers accompanying the drop deformation might play a role. Figure 11 shows that the temperature used to calculate the liquid properties turns out to only have a marginal effect on the amplitude of drop spreading.

Figures 10 and 11 show that the acceleration of the rim is not high enough in the model predictions. Also, the fact that the differences vanish for the high viscous liquids supports the idea that there is an additional force which pulls the liquid in the lamella along the radial direction. The assumption that the vapor released by the drop could cause a shear stress at the liquid surface, ease the liquid slip and thus lead to higher spreading has already been proposed. ${ }^{16,40}$ Due to the small thickness of the vapor layer between the wall and the droplet (measured at a few micrometers by van der Veen et $a l .{ }^{41}$ ), it is understandable that the vapor flow can reach relatively high velocities and transfer some momentum to the liquid inside the drop. Tran et al.${ }^{16}$ carried out a scaling analysis to estimate the maximum spreading by balancing the shear stress at the liquid/vapor interface and the capillary force. This yields $\beta_{\max } \sim W e^{0.3}$. However, this scaling does not provide a perfect match with the experimental observations. In the following, the radial velocity $u_{\text {rim }}$ of the liquid entering the rim is replaced by

$$
u_{\text {rim }}^{*}=\frac{(1+\alpha) r^{*}}{t^{*}+\tau} \text { at } r^{*}=R_{R}^{*}
$$

where $\alpha$ is a factor corresponding to the increase in velocity of the flow entering the rim. Fixing $\alpha>0$ is expected to compensate for the lack of momentum transferred to the rim. The introduction of this parameter requires certain modifications to the forces acting on the rim,

$$
F_{i l R}^{*}=\left(\dot{R}_{R}^{*}-\frac{(1+\alpha) R_{R}^{*}}{t^{*}+\tau}\right)^{2} \text { and } F_{v}^{*}=\frac{6(1+\alpha)}{\operatorname{Re}\left(t^{*}+\tau\right)} .
$$

For the rest of Sec. VI, we adjust the value of the parameter $\alpha$ to obtain the best match between the model and the experiments. Besides the fact that $\alpha$ allows the initial model described in Sec. IV
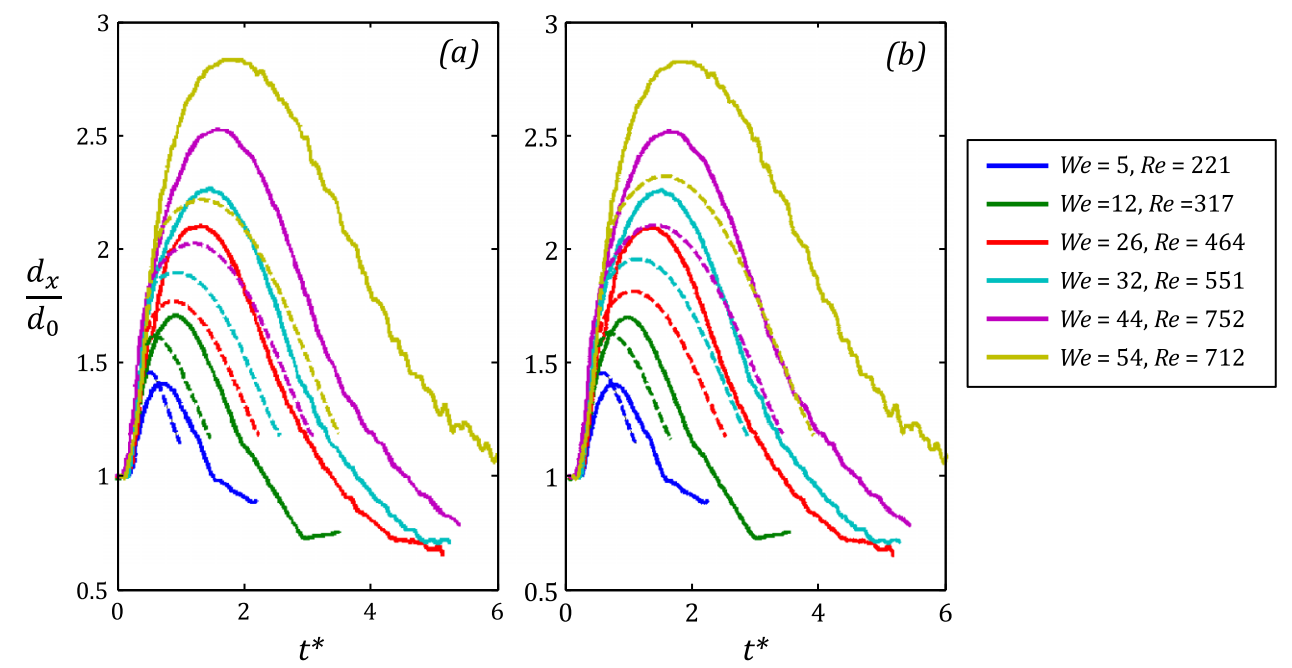

FIG. 11. Time evolution of the spreading factor $\beta=d_{x} / d_{0}$ of water drops. Predictions of the model assuming two temperatures of the liquid: (a) $T=23^{\circ} \mathrm{C}$ and (b) $T=100^{\circ} \mathrm{C}$. The solid lines: experimental data from the same cases as in Fig. 10(a); the dotted lines: solutions of Eq. (16) assuming the inviscid flow solution (Eq. (8) with $A=1$ ) to calculate the velocity of the liquid entering the rim. 
to be corrected, the question arises as to the physical meaning of this parameter. This question will be addressed in more detail in Sec. VII, where it will be established that this parameter can be related to the main features of the entrainment boundary layer that develops at the bottom edge of the lamella due to the action of the vapor flow.

In the following calculations, the thickness of the lamella is still evaluated with Eq. (10). However, if the liquid is dragged by the vapor film, the lamella thickness will change in consequence. An entrainment boundary layer grows over time and eventually compares with the lamella thickness. An estimate of this boundary layer thickness will be given in Sec. VII. At the initial stage of the spreading, it is a good approximation to consider the lamella thickness at $r=R_{R}$ to be roughly equal to Eq. (10).

Figure 12 gives the predictions of the theoretical model with the adjustment of $\alpha$ for the cases already presented in Fig. 10. The adjustment of the correction factor $\alpha$ provides a rather good match between the model and experimental data. The model seemingly properly captures both the spreading amplitude and the time of maximum spreading $t_{x, \max }$. In Fig. 13, $t_{x, \max }$ appears to be about the same in both the experiments and calculations. In this figure, $t_{x, \max }$ and $t_{c}$ (the contact time) are normalized by the free oscillations period of the drop $t_{o s c}=\pi / 4\left(\rho d_{0}^{3} / \sigma\right)^{1 / 2}$, since the contact time is known to be of the same order as $t_{o s c} .{ }^{5}$ The spreading time $t_{x, \max }$ behaves approximately as $t_{o s c} / 3$ with little difference between the liquids. At $W e<10$, the spreading phase lasts proportionally longer than $t_{o s c} / 3$ and this trend is also reproduced by the model.

Figure 14 shows the results of tests on several values of $\alpha$ which were run to determine the level of confidence in the estimate of $\alpha$. This figure shows for different impact conditions that the factor $\alpha$ can be estimated with uncertainty of the order of 0.05 when it is adjusted to obtain the best match between the experimental data and the theoretical model. This level of uncertainty is slightly greater for the low Weber numbers.
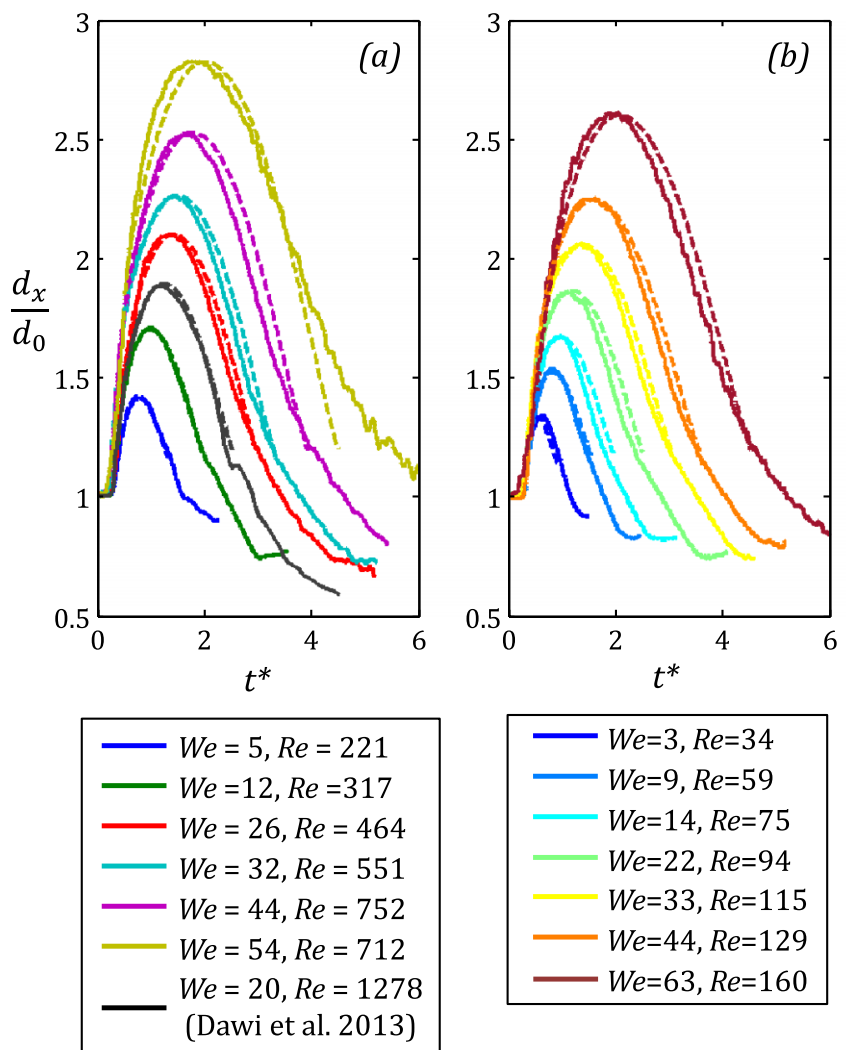
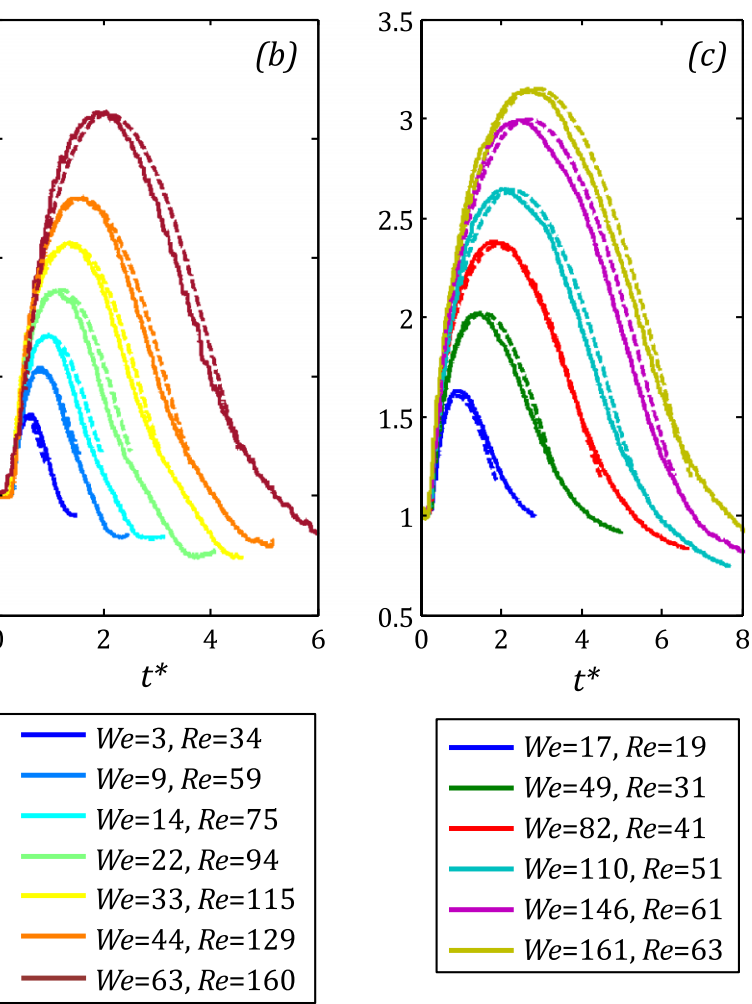

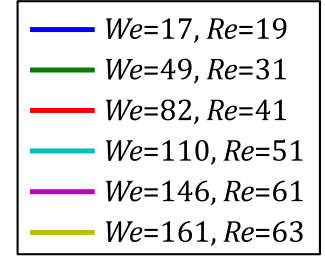

FIG. 12. Time evolution of the spreading factor $\beta=d_{x} / d_{0}$ for several Weber numbers and three liquids: (a) water, (b) mixture of $44 \%$ glycerol $/ 56 \%$ water, (c) mixture of $70 \%$ glycerol $/ 30 \%$ water. The impact conditions are the same as in Fig. 10. Solid lines: experimental data; dotted lines: solutions of Eq. (16) while adjusting the factor $\alpha$ in Eq. (20). 


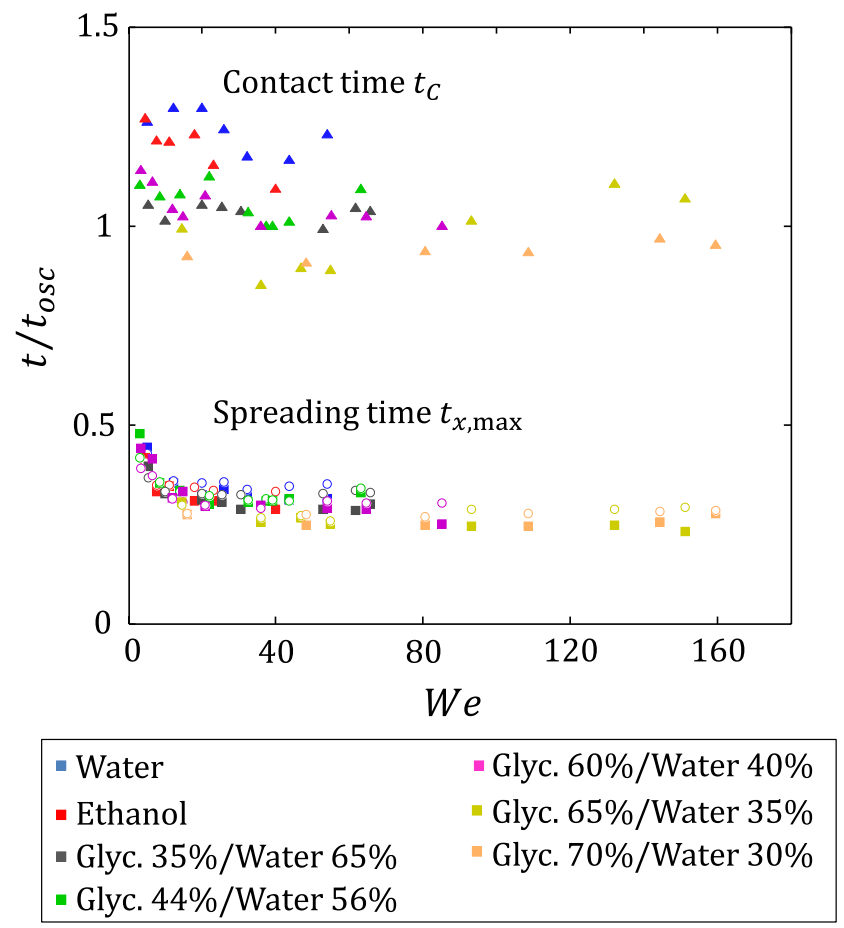

FIG. 13. Dependence of the contact time $t_{c}$ and the spreading time $t_{x, \max }$ on the Weber number. Times are normalized by the free oscillation period $t_{o s c}$. Squares: measurements of $t_{x, \max }$; hollow circles: values of $t_{x, \max }$ calculated from the model while adjusting the factor $\alpha$ in Eq. (20); triangles: measurements of $t_{c}$.

The adjusted values of $\alpha$ obtained for the full set of impact conditions tested in the experiments are presented in Fig. 15. For a given liquid, the parameter $\alpha$ decreases with the Weber number and eventually reaches a plateau which means that the factor $\alpha$ does not depend on the impact conditions for sufficiently high Weber and Reynolds numbers. The value of $\alpha$ at the plateau only depends on the nature of the liquids, the largest value being obtained for the least viscous liquid. This value is about 0.1 for the most viscous mixtures of water and glycerol, while it equals about 0.47 for water.

Outside the plateau (i.e., for the low Weber and Reynolds numbers), $\alpha$ is found to be clearly dependent on the impact conditions as its value rapidly decreases with $W e$. Any effect of liquid viscosity can be hardly pointed out in this domain. The adjusted values of $\alpha$ are all distributed around

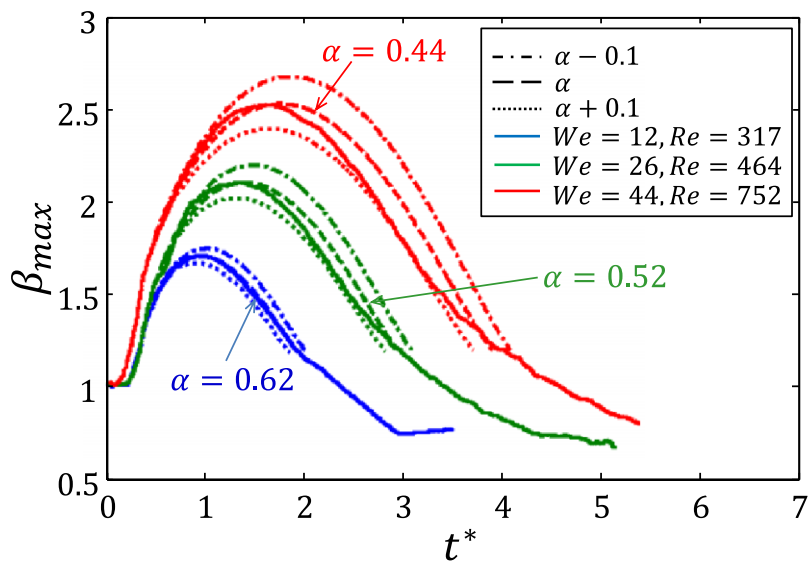

FIG. 14. Sensitivity of the model to the value of the factor $\alpha$ in Eq. (20). Case of water drops and three different Weber numbers. Solid lines: experimental data; dotted and dashed lines: model predictions. 


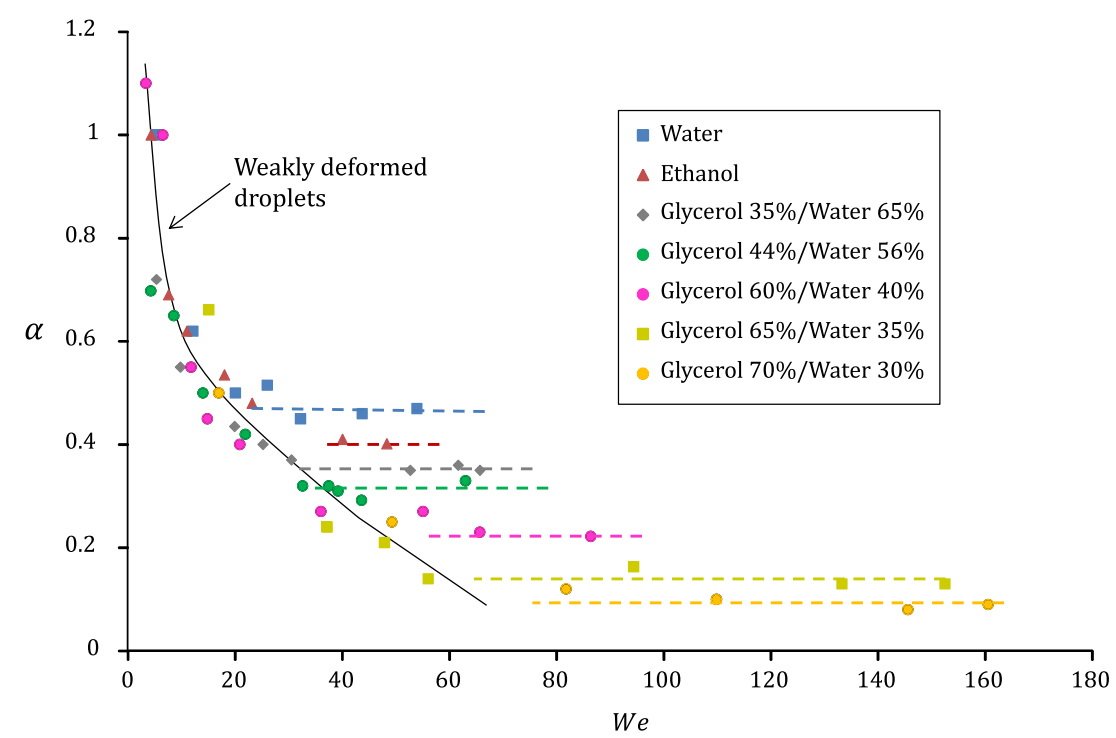

FIG. 15. The influence of the Weber number on the adjusted value of $\alpha$ in Eq. (20). Points: adjustments of $\alpha$ based on the experimental results; the solid line: fitted curve corresponding to droplets weakly deformed at the impact; dotted lines: estimated values of $\alpha$ at high Weber and Reynolds numbers.

the black curve plotted in Fig. 15. For these droplets impacting at low $R e$ and $W e$, the deformation is rather weak and the theoretical model is not used within its range of validity. Equations (8) and (10), which provide the flow field inside the lamella and the thickness distribution of the lamella, are valid at high Reynolds and Weber numbers. If these numbers are too small, the simplifications used to infer Eqs. (8) and (10) do not hold, meaning that the deformation of the drop is governed by the viscous stresses and surface tension alongside the inertial forces. The conditions for Eqs. (8) and (10) have been discussed in more detail by Roisman et al. ${ }^{21}$ A comparison between the two thicknesses can be found in Figs. 13 and 14 in Roisman et al. ${ }^{21}$ which clearly show that the droplet height for the low $R e$ is always higher than approximation (10), especially near the edge of the droplet. Therefore, one explanation for the high values of $\alpha$ observed at low $W e$ in Fig. 15 may be that $\alpha$ is also increased to compensate for the insufficient thickness given by Eq. (10). When We (and also $R e$ since $O h$ is almost a constant for a given liquid within the sets of measurements) is increased, approximations (8) and (10) become valid as the plateau is reached. Roisman et al. ${ }^{21}$ found that the flow inside the lamella and the shape evolution of the lamella do not depend on the impact conditions provided sufficiently high $R e$ and $W e$. Hence, it seems that the factor $\alpha$ becomes independent of impact conditions when the lamella evolution (velocity field and shape) does likewise.

Figure 16 displays the variation of $\beta_{\max }$ predicted by the theoretical model as $R e$ varies over four orders of magnitude while $\alpha$ retains its value at the plateau. In Fig. 16, $\alpha$ is set equal to 0.47 which corresponds to the case of water drops. $\beta_{\max }$ can be observed to not vary a great deal with the Reynolds number provided that $R e>100$. Hence, the model predicts that two water droplets with different diameters (respectively, $100 \mu \mathrm{m}$ and $2 \mathrm{~mm}$ ) and the same $W e$ will have the same maximum spreading factor $\beta_{\max }$ (Fig. 16). This prediction perfectly matches with the experimental observations shown in Fig. 9 whereby the droplet size has no influence on $\beta_{\max }$ as droplets vary in size from about $100 \mu \mathrm{m}$ to a few millimeters.

Again for $\alpha=0.47$, the model is used to simulate the spreading of $2 \mathrm{~mm}$-size water drops as $W e$ varies from 15 to 240 . To perform these simulations, the initial conditions are fixed at $t_{i}^{*}=$ 0.5 as $R_{R}^{*}\left(t_{i}^{*}\right)=0.7$ and $\dot{R}_{R}^{*}\left(t_{i}^{*}\right)=0.7$. Reminding that $R_{R} \approx d_{c} / 2$, these values are very close to $R_{R}^{*}\left(t_{i}^{*}\right)=2 \sqrt{t_{i}^{*}}$ and $\dot{R}_{R}^{*}\left(t_{i}^{*}\right)=R_{R}^{*}\left(t_{i}^{*}\right) /\left(2 t_{i}^{*}\right)$ which are known features of the kinematic phase. They also prove to be well adapted to the simulation of our experiments. Figure 17 shows a perfect agreement with the experimental data obtained by Tran et al. ${ }^{7}$ up to Weber numbers on the order of 200 . Hence, a unique value of $\alpha$ (presently 0.47) can be successful in describing the spreading of water drops with a diameter of a few millimeters for Weber numbers ranging from 7 to 200. This range of 


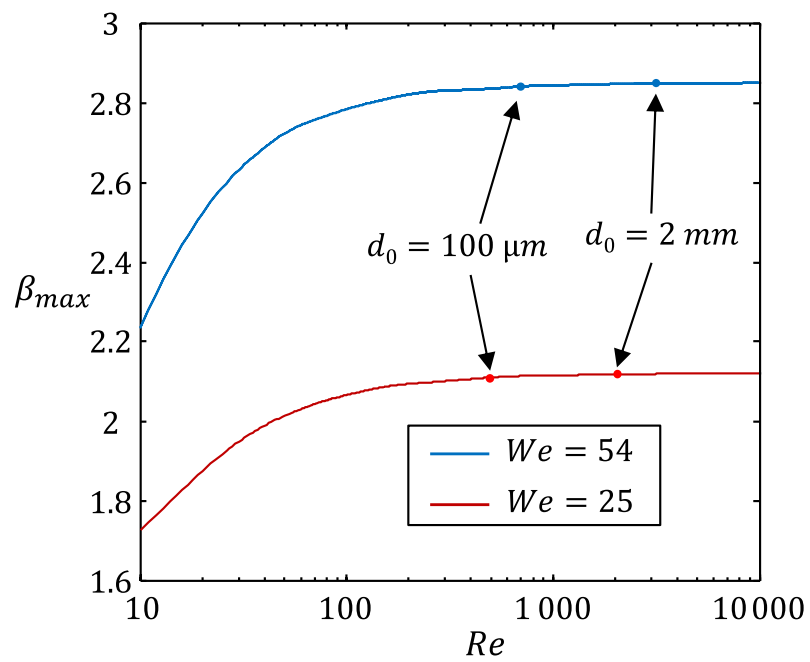

FIG. 16. Influence of the Reynolds number on the maximum spreading factor $\beta_{\max }$ evaluated from the theoretical model when $\alpha$ is equal to 0.47 . Indicated values of $d_{0}$ are given assuming a water droplet.

$W e$ is a little larger than for the droplets with a diameter of about $100 \mu \mathrm{m}$ (Fig. 15) which is probably due to the fact that the high $R e$ approximation is easier to satisfy for the large droplets at low $W e$.

Figure 18 shows that the values of $\alpha$ obtained at high $W e$ correlate well with the kinematic viscosity of the liquid $v$. The parameter $\alpha$ appears to be a function of the liquid kinematic viscosity without directly being a function of the Reynolds number. In fact, the viscous effects highlighted in Fig. 8 for the maximum spreading diameter are essentially included in the adjustment of $\alpha$. It should be noted that $v^{-1 / 5}$ is also an acceptable (and in fact even better) scaling for the maximum spreading factor in Fig. 8. As a partial conclusion, the previous observations concerning the correction factor $\alpha$ do not contradict the idea that the lamella could be pulled radially by the vapor flow below the droplet. As expected for an entrainment by an external flow, the value of $\alpha$ is found to be positive and to decrease mainly with the liquid viscosity. It tends to 0 at high liquid viscosity. Also, the

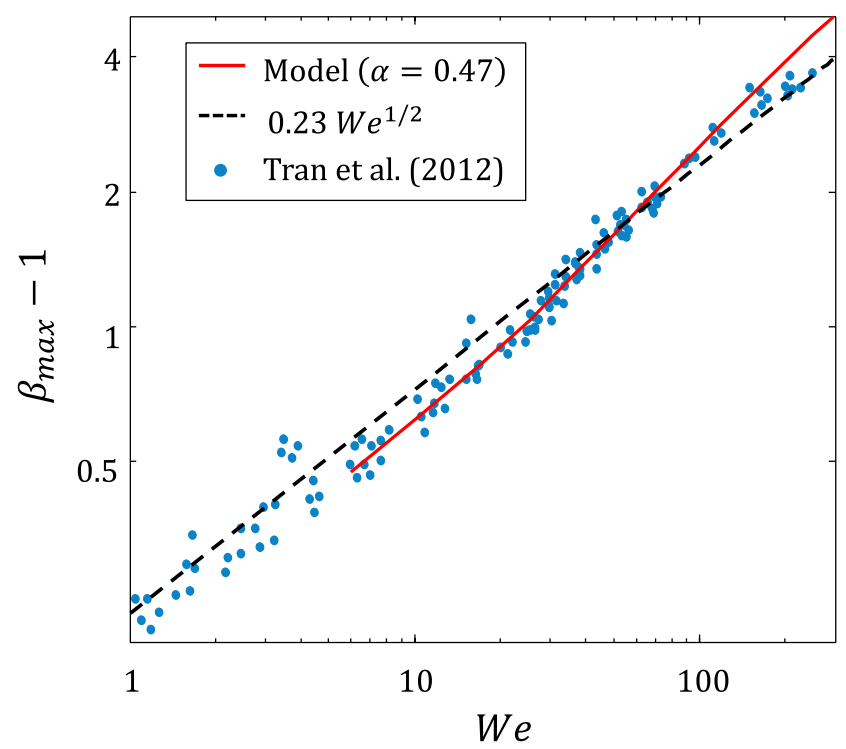

FIG. 17. Evolution of the maximum spreading factor $\boldsymbol{\beta}_{\max }$ as a function of the Weber number in the case of water droplets. Comparison between the measurements by Tran et al. ${ }^{7}$ and the model based on Eq. (16). Solid line: predictions of the model fixing $\alpha=0.47$ and $d_{0}=2 \mathrm{~mm}$; the dotted line: fitted curve $0.23 W e^{1 / 2}$ introduced in Fig. 9; points: experimental data of Tran et al. ${ }^{7}$ corresponding to water drops sizing about $2 \mathrm{~mm}$. 


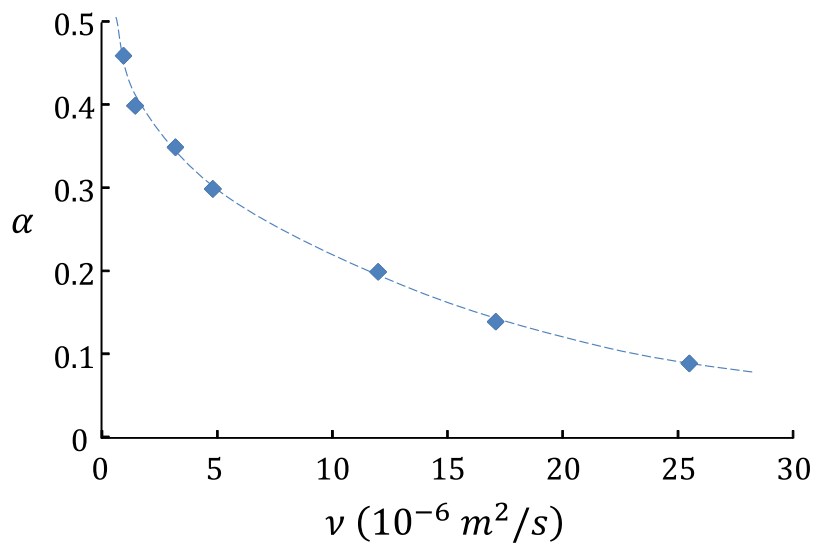

FIG. 18. Influence of the liquid viscosity on the adjusted value of $\alpha$ at high Weber and Reynolds numbers. Each point corresponds to one of the liquids tested in the experiments. Solid line: fitted curve.

factor $\alpha$ is not influenced by the impact conditions provided $W e$ and $R e$ are sufficiently large. Still, it remains to establish a more direct link between the correction factor $\alpha$ and the entrainment boundary layer that develops at the bottom edge of the lamella (Fig. 19).

\section{MODELING OF THE LAMELLA ENTRAINMENT BY THE VAPOR FLOW}

In this section, we consider the action of the vapor film upon the lamella. Modeling of the vapor film and its action upon the spreading of the impacting drop is a problem which remains to be solved. Certain simplifying assumptions are required to be made. The approach described below is adapted from existing analyses of the sessile drop evaporation in Leidenfrost boiling. ${ }^{42-44}$ In particular, it is based on the lubrication approximation applied to the vapor film, the thickness of which is assumed to be uniform but to vary in time. It is critical for such modeling to account for the continuity of the shear stress at the liquid/vapor interface (Fig. 19) to evaluate the capability of the vapor to pull the lamella along the radial direction.

In the drop impact in the Leidenfrost conditions, the vapor film below the droplet is dramatically squeezed because of the impact pressure. The impact pressure exceeds by far the static pressure related to the weight of the liquid puddle on the top of it. At the very beginning of the impact, the impact pressure is the strongest below the truncated sphere already mentioned in the

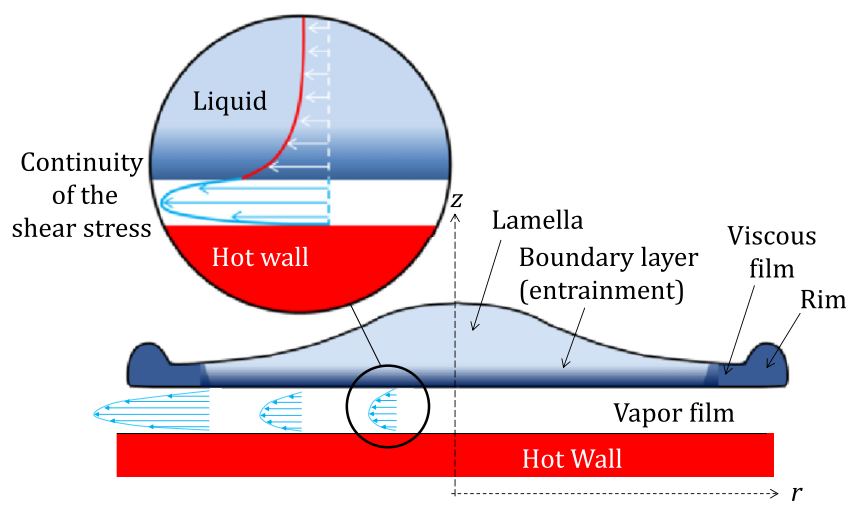

FIG. 19. Development of an entrainment boundary layer at the bottom edge of the lamella. Given that the vapor has a radial velocity much higher than that of the liquid in the lamella, the vapor flow exerts a shear stress on the liquid interface and induces the formation of an entrainment boundary layer in the lamella. 
description of the kinematic phase of the drop spreading. The pressure rapidly decreases with time as shown by Roisman et al. ${ }^{21}$ who numerically found an exponential decay at the axis of symmetry $(r=0)$. As a consequence of the high pressure exerted by the drop at the impact, the vapor layer is much thinner than in the case of a gently deposited sessile drop. The vapor film thickness typically differs by two orders of magnitude between the two situations. ${ }^{40}$ Recent measurements by van der Veen et al. ${ }^{41}$ found that the thickness of the vapor film under a water drop of a few millimeters at a low Weber number $(W e=3.5)$ can be as small as $3 \mu \mathrm{m}$ but these measurements were taken just after the beginning of the impact (when the thickness is near its minimum). Drop impacts involve a thinner layer of vapor which promotes a higher evaporation rate and a larger velocity of the vapor flow. Also, an impacting drop spreads over a larger surface which contributes to a higher velocity being reached in the vapor film. Obviously, another major difference with a sessile droplet is that the droplet deformation is strongly influenced by inertia and transient effects as previously described in Sec. IV.

If we assume the vapor flux under the bottom edge of the droplet to be uniform, then the mean radial velocity $u_{v}(r, t)$ in the vapor layer can be determined by

$$
u_{v}(r, t)=\frac{\dot{m}_{v}^{\prime \prime} r}{2 \rho_{v} \delta_{v}}
$$

where $\rho_{v}$ is the vapor density and $\dot{m}_{v}^{\prime \prime}=4 \dot{m}_{v} / \pi \beta^{2} d_{0}^{2}$ is the mass flow rate of vapor per unit of surface. In addition to the mass flux $\dot{m}_{v}^{\prime \prime}$, the thickness $\delta_{v}$ of the vapor film is also supposed to be uniform under the drop. Equation (22) is derived from the application of mass conservation to the vapor between radial distances of 0 and $r$. As the parameters $\beta, \delta_{v}$, and $\dot{m}_{v}^{\prime \prime}$ are time varying, Eq. (22) should be regarded as a quasi-steady approximation which is clearly valid for a sessile drop but a little more disputable in the case of an impact. However, it is likely that quasi-stationary conditions are rapidly established in the vapor film. After an initial phase of rapid change, the thickness of the vapor film varies very slowly in comparison with the vapor residence time under the drop. ${ }^{45}$ Also, it should be emphasized that the velocities of the vapor (22) and the liquid in the lamella (8) are both proportional to the radial distance $r$.

Given the very small thickness of the vapor layer, heat is predominantly transferred from the hot wall to the drop through heat conduction. The corresponding heat flux can be expressed as

$$
\varphi=\lambda_{v}\left(T_{w}-T_{s a t}\right) / \delta_{v},
$$

where $\lambda_{v}$ is the thermal conductivity of the vapor and $T_{\text {sat }}$ denotes the saturation temperature of the liquid. The heat transferred from the wall to the droplet is used both to heat the liquid and for its vaporization at the droplet surface. A sessile drop remains a sufficient time close to the wall with the result that the whole liquid volume reaches $T_{s a t}$. As a result, $\varphi \approx L_{v} \dot{m}_{v}^{\prime \prime}$, where $L_{v}$ is the latent heat of vaporization of the liquid. In the case of a drop impact, the transient heating of the droplet cannot be ignored given that the sensible heat flux entering the droplet usually compares with the heat flux of vaporization as shown in recent experiments by Dunand et al..$^{38}$

The geometry of the vapor film (a thin and long slot) naturally sets the conditions for the lubrication approximation meaning that the vapor escapes radially with a Poiseuille flow. Assuming a no-slip condition at the solid and liquid interfaces (the smallness of the slip velocity at the vapor/liquid interface in comparison with the mean vapor velocity can be checked afterwards), the viscous stress at the interface can be evaluated as $\tau_{v}=6 \mu_{v} u_{v} / \delta_{v}$ which yields

$$
\tau_{v}(r)=3 \dot{m}_{v}^{\prime \prime} v_{v} r / \delta_{v}^{2}
$$

where $v_{v}=\mu_{v} / \rho_{v}$ is the kinematic viscosity of the vapor.

In the spreading liquid film, a boundary layer develops at the bottom edge of the droplet (Fig. 19). It is possible to account for this boundary layer by introducing a scaled stream function $g(\xi)$ and the self-similar variable $\xi=z / \sqrt{v t}$,

$$
u_{r}=g^{\prime}(\xi) \frac{r}{t}, \quad u_{z}=-2 g(\xi) \sqrt{v / t}
$$


The stream function $g$ needs to satisfy the momentum balance equation in the radial direction for an axisymmetric flow in the absence of pressure gradient and body forces,

$$
\frac{\partial u_{r}}{\partial t}+u_{r} \frac{\partial u_{r}}{\partial r}+u_{z} \frac{\partial u_{r}}{\partial z}=v\left\{\frac{\partial}{\partial r}\left[\frac{1}{r} \frac{\partial\left(r u_{r}\right)}{\partial r}\right]+\frac{\partial^{2} u_{r}}{\partial z^{2}}\right\} .
$$

Substituting Eq. (25) into (26), the following ordinary differential equation can be obtained: ${ }^{24}$

$$
g^{\prime \prime \prime}+2 g g^{\prime \prime}+\frac{1}{2} \xi g^{\prime \prime}+g^{\prime}-g^{\prime 2}=0,
$$

with the associated boundary conditions: $g=0$ and $g^{\prime}=g^{\prime}(0)$ at $\xi=0$ and $g^{\prime}=1$ at $\xi \rightarrow \infty$. Based on Eq. (25), the viscous stress at the liquid/vapor interface can be determined by

$$
\tau_{l}=g^{\prime \prime}(0) \sqrt{v} \rho r t^{-3 / 2} .
$$

Considering the continuity of the shear stress at the liquid-vapor interface $\left(\tau_{v}=\tau_{l}\right)$, Eqs. (24) and (28) yield

$$
g^{\prime \prime}(0)=\frac{3 \dot{m}_{v}^{\prime \prime} v_{v}}{\rho \sqrt{v} \delta_{v}^{2}} t^{3 / 2}
$$

Equation (27) has to be solved numerically. The value of $g^{\prime}(0)$ is a priori unknown. It is determined iteratively by using a standard shooting method to satisfy the condition $g^{\prime}(\xi)=1$ at $\xi \rightarrow \infty$. This means the slip velocity of the liquid/vapor interface can then be found using Eq. (25).

Reminding that $u_{r}=r /\left(t+\tau_{0}\right)$ corresponds to the inviscid flow solution outside the boundary layer, the cross sectional average velocity $u_{\text {rim }}$ of the liquid entering the rim can be approximated by

$$
u_{\text {rim }} \approx \frac{R_{R}}{t+\tau_{0}}+\frac{\delta_{2}}{h\left(R_{R}\right)} \cdot \frac{g^{\prime}(0) R_{R}}{t+\tau_{0}} .
$$

In this expression, $\delta_{2}$ corresponds to the momentum thickness of the boundary layer. By definition, $\delta_{2}=\gamma \sqrt{v t}$ with $\gamma=\int_{0}^{1} g^{\prime}(\xi)\left[1-g^{\prime}(\xi)\right] d \xi$. Equation (30) implies that the boundary layer is much thinner than the lamella $\left(h \gg \delta_{2}\right)$. Otherwise, the condition $g^{\prime}(\xi)=1$ may not be satisfied at the free surface on the top of the lamella, which would invalidate the boundary-layer analysis which was carried out. By comparing Eqs. (20) and (30), an expression for the correction factor $\alpha$ introduced in Sec. VI can be obtained as

$$
\alpha=\frac{\delta_{2}}{h\left(R_{R}\right)} g^{\prime}(0) .
$$

Equation (31) can be used to successfully estimate the value of $\alpha$ despite certain assumptions. However, the major concern with the application of Eq. (31) is that it requires perfect knowledge of the vapor layer thickness, and determining this remains a challenging task in the case of a drop impact. To the best of our knowledge, approaches in the literature mainly involve scaling analyses which only provide rough estimates of the vapor film thickness. Also as these analyses generally deal with sessile drops, they miss some of the transient aspects which are critical in drop impacts. These include particularly the transient impact pressure between the droplet and the transient heating of the liquid which may have a considerable effect on the thickness of the vapor layer. In the absence of a theoretical model for the vapor thickness below the droplet, Eq. (31) will be assessed using the results provided by the direct numerical simulation of Dawi et al..$^{45}$ whose calculations concerned water droplets sizing $1 \mathrm{~mm}$ which impact onto a surface heated at $T_{\mathrm{w}}=350{ }^{\circ} \mathrm{C}$. Dawi et al. ${ }^{45}$ obtained numerical results in good agreement with the experiments run by Tran et al. ${ }^{7}$ Their simulated evolution of the spreading factor $\beta=d_{x} / d_{0}$ at $W e=20$ is reproduced well by the present model of drop spreading (with $\alpha=0.47$ ) as displayed in Fig. 12. Also, Fig. 20 shows a good agreement for the droplet shape at different times of the spreading.

The evaporation rate $\dot{m}_{v}$, the vapor thickness $\delta_{v}$, and the spreading factor $\beta$ are provided by the numerical simulation of Dawi et al. ${ }^{45}$ At $t^{*}=1$, the simulation gives $\dot{m}_{v}=2.9 \cdot 10^{-7} \mathrm{~kg} / \mathrm{s}$, 


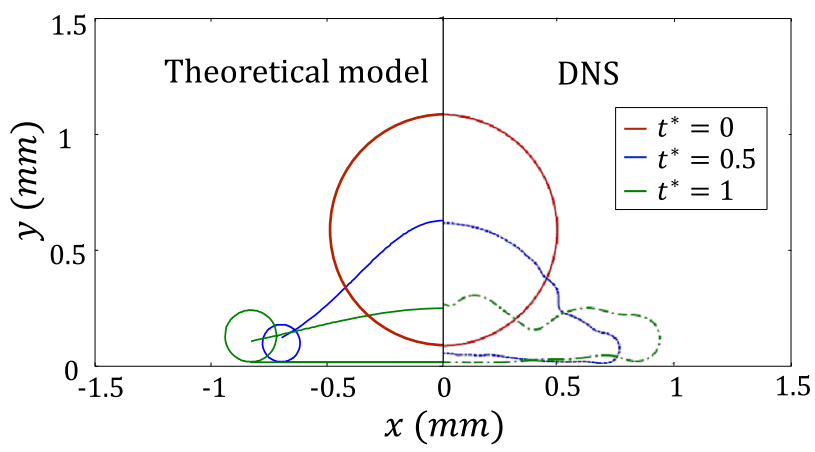

FIG. 20. Evolution of the droplet shape during the spreading phase. Comparison between the theoretical model (solid lines) and Direct Numerical Simulation (DNS) by Dawi et al. ${ }^{45}$ (dotted lines) at different times. Case of a water drop $\left(d_{0}=1 \mathrm{~mm}, W e=20\right) . \alpha$ is fixed at 0.47 in the calculations.

$\delta_{v} \approx 15 \mu \mathrm{m}$, and $\beta \approx 1.89$. Using Eq. (22), this leads to the following estimates of the cross sectional velocity: $u_{v}[\mathrm{~m} / \mathrm{s}] \approx 8200 r[\mathrm{~m}]$. It should be noted that $\delta_{v}$ remains roughly uniform and about equal to $15 \mu \mathrm{m}$ in the simulation during most of the spreading phase beyond $t^{*}=0.5$. This argues in favor of the validity of the quasi-steady approximation upon which Eq. (22) is based. The shear stresses at the liquid/vapor interface can be determined using Eqs. (24) and (29): $\tau_{v}\left[\mathrm{~N} \cdot \mathrm{m}^{-2}\right]=59789 r[\mathrm{~m}]$ and $g^{\prime \prime}(0)=3.11$. For these calculations, the physical properties of the vapor are evaluated at the film temperature $\frac{T_{w}+T_{s a t}}{2}=225^{\circ} \mathrm{C}$. For the liquid phase, the properties of water are taken at the boiling temperature $\left(\rho=958 \mathrm{~kg} \cdot \mathrm{m}^{-3}, v=2.94 \cdot 10^{-7} \mathrm{~m}^{2} \cdot \mathrm{s}^{-1}\right.$, $\rho_{v}=0.42 \mathrm{~kg} \cdot \mathrm{m}^{-3}$, and $\left.\mu_{v}=18.3 \cdot 10^{-6} \mathrm{~Pa} \cdot \mathrm{s}\right)$. A Runge-Kutta scheme is used to solve Eq. (27) with the constraint that $g(0)=0$ and $g^{\prime \prime}(0)=3.11$. The value of the slip velocity $g^{\prime}(0)$ is found to be equal to 2.45 to satisfy the condition $g^{\prime}(\xi)=1$ at $\xi \rightarrow \infty$. Based on the solution obtained for $g(\xi)$, the momentum thickness of the boundary layer $\delta_{2}=\gamma \sqrt{v t}$ can be estimated. At $t^{*}=1$, values of $\gamma=0.93$ and $\delta_{2}=15 \mu \mathrm{m}$ are obtained. This thickness needs to be compared with the height of the lamella. The model of drop spreading (16) predicts a rim position $R_{R}=0.825 \mathrm{~mm}$ while $h\left(R_{R}\right)=90 \mu \mathrm{m}$ using thickness profile (10). Hence, the ratio $\delta_{2} / h\left(R_{R}\right)$ is small and Eq. (30) can be considered an acceptable approximation for the velocity $u_{\text {rim }}$ of the liquid entering the rim. Finally, the application of Eq. (31) leads to $\alpha=0.41$, which is not far from the figure of 0.47 obtained from the experimental data in Sec. VI. Running the same calculations on another time during the spreading phase yield relatively different values of $\alpha$ (about 0.31 and 0.57 for $t^{*}=0.7$ and $t^{*}=1.2$, respectively). The factor $\alpha$ increases over time during the spreading, as the entrainment boundary layer develops and the thickness of the lamella decreases. These estimates of $\alpha$ confirm the importance of the action of the vapor upon the sliding velocity of the liquid interface and the spreading of the drop despite approximations and assumptions made in the evaluation of $\alpha$. For example, in Fig. 20, the drop thickness appears to be slightly larger near the edge of the lamella in the numerical simulation by Dawi et al. ${ }^{45}$ about $120 \mu \mathrm{m}$ against $90 \mu \mathrm{m}$ at $t^{*}=1$. Using this value of the lamella thickness in Eq. (31) would yield $\alpha=0.31$. Here also, the vapor flow still significantly increases the slip velocity of the liquid/vapor interface which leads to a larger spreading of the droplet.

\section{CONCLUSION}

Most advanced models concerning drop spreading consider a non-dissipative flow in the inside of the lamella, provided that Reynolds and Weber numbers are sufficiently high (typically more than a few tens). The consequence of this description is that viscous dissipation would not occur in the inside of the lamella but instead at the bottom edge of the lamella where frictions are exerted on the spreading droplet. With Leidenfrost boiling, the question of liquid viscosity influence on the spreading process arises because the kinematic condition at the interface between the liquid and the vapor film is not well defined. The assumption of perfect sliding at the liquid/vapor interface has the advantage of being easy to formulate. Mass and momentum balances applied to the rim 
bounding the lamella provide an ordinary differential equation for the rim motion which can be solved numerically. The model accounts for the forces acting on the rim: the momentum transferred by the liquid entering the rim, the surface forces, and the viscous stress at the surface separation between the rim and the lamella. Although the flow retained in the model for the lamella corresponds to the inviscid flow of the remote asymptotic solution, the model predictions systematically underestimate droplet deformation and are not capable of capturing the experimental dependence of the maximum spreading factor on the liquid viscosity. However, measurements of the lamella thickness near the center of the droplet match well with the inverse square law with time implied by this flow solution. Combining these facts leads to the assumption that the liquid motion inside the lamella is accelerated radially by the vapor flow. The small thickness of the vapor layer means that the vapor flow under the droplet can reach relatively high velocity and induce significant shear stress on the liquid surface. The thickness of the resulting boundary layer remains negligible compared to the lamella in the central region of the droplet but can reach about the same thickness near the edge of the lamella leading to an increase of mass and momentum transferred to the rim. If the velocity of the liquid entering the rim is artificially increased in the model, remarkable agreements can be obtained between the experiments and the simulations. In the case of water, the increase is about $50 \%$ which reflects the significance of the liquid drag-out. For more viscous liquids, the increase is more limited and asymptotically tends to 0 with the liquid viscosity. Finally, the assumption that the liquid motion is accelerated by the vapor film has been assessed based on a modeling of the entrainment boundary layer under lubrication approximation applied to the vapor flow. Numerical applications rely on the results of a direct numerical simulation of the drop spreading at the impact in the Leidenfrost boiling. The shear stress at the liquid/vapor interface, and thus the thickness of the boundary layer at the bottom edge of the lamella, is evaluated from these numerical simulations. The results confirm that there is a substantial increase in liquid velocity at the rim entrance.

\section{ACKNOWLEDGMENTS}

The authors are very grateful to I. Roisman for fruitful discussions and exchange of data. The authors also acknowledge the financial support of l'Agence Nationale de la Recherche (ANR) through Grant No. ANR-NT09 432160 (ANR “IDHEAS”).

${ }^{1}$ D. M. A. Gonzalez, G. L. Borman, and R. D. Reitz, A Study of Diesel Cold Starting using both Cycle Analysis and Multidimensional Calculations (Society of Automotive Engineers, New York, NY, USA, 1991).

2 J. D. Bernardin and I. Mudawar, "Film boiling heat transfer of droplet streams and sprays," Int. J. Heat Mass Transfer 40, 2579 (1997).

${ }^{3}$ R. Rioboo, C. Tropea, and M. Marengo, "Outcomes from a drop impact on solid surfaces," Atomization Sprays 11, 12 (2001).

${ }^{4}$ A. Karl and A. Frohn, "Experimental investigation of interaction processes between droplets and hot walls," Phys. Fluids 12, 785 (2000).

${ }^{5}$ L. H. J. Wachters and N. A. J. Westerling, "The heat transfer from a hot wall to impinging water drops in the spheroidal state," Chem. Eng. Sci. 21, 1047 (1966).

${ }^{6}$ N. Hatta, H. Fujimoto, K. Kinoshita, and H. Takuda, "Experimental study of deformation mechanism of a water droplet impinging on hot metallic surfaces above the Leidenfrost temperature," J. Fluids Eng. 119, 692 (1997).

${ }^{7}$ T. Tran, H. J. J. Staat, A. Prosperetti, C. Sun, and D. Lohse, “Drop impact on superheated surfaces," Phys. Rev. Lett. 108, 036101 (2012).

${ }^{8}$ A.-L. Biance, F. Chevy, C. Clanet, G. Lagubeau, and D. Quéré, “On the elasticity of an inertial liquid shock,” J. Fluid Mech. 554, 47 (2006).

${ }^{9}$ A.-L. Biance, C. Pirat, and C. Ybert, "Drop fragmentation due to hole formation during Leidenfrost impact," Phys. Fluids 23, 022104 (2011).

${ }^{10}$ N. G. Rosa, P. Villedieu, J. Dewitte, and G. Lavergne, "A new droplet-wall interaction model," in 10th International Conference on Liquid Atomization and Spray Systems, Kyoto, Japan, August 27-September 1, 2006.

${ }^{11}$ J. D. Bernardin and I. Mudawar, "A Leidenfrost Point model for impinging droplets and sprays," J. Heat Transfer 126, 272 (2004).

12 S.-C. Yao and K. Y. Cai, "The dynamics and Leidenfrost temperature of drops impacting on a hot surface at small angles," Exp. Therm. Fluid Sci. 1, 363 (1988).

${ }^{13}$ D. Bartolo, C. Josserand, and D. Bonn, "Retraction dynamics of aqueous drops upon impact on non-wetting surfaces," J. Fluid Mech. 545, 329 (2005).

${ }^{14}$ Š. Šikalo, M. Marengo, C. Tropea, and E. N. Ganić, "Analysis of impact of droplets on horizontal surfaces," Exp. Therm. Fluid Sci. 25, 503 (2002).

${ }^{15}$ C. Antonini, I. Bernagozzi, S. Jung, D. Poulikakos, and M. Marengo, "Water drops dancing on ice: How sublimation leads to drop rebound," Phys. Rev. Lett. 111, 014501 (2013). 
${ }^{16}$ T. Tran, H. J. J. Staat, A. Susarrey-Arce, T. C. Foertsch, A. van Houselt, H. Gardeniers, A. Prosperetti, D. Lohse, and C. Sun, "Droplet impact on superheated micro-structured surfaces," Soft Matter 9, 3272 (2013).

${ }^{17}$ S. Chandra and C. T. Avedisian, "On the collision of a droplet with a solid surface," Proc. R. Soc. London, Ser. A 432, 13 (1991).

${ }^{18}$ T. Mao, D. C. S. Kuhn, and H. Tran, "Spread and rebound of liquid droplets upon impact on flat surfaces," AIChE J. 43, 2169 (1997).

${ }^{19}$ M. Pasandideh-Fard, Y. M. Qiao, S. Chandra, and J. Mostaghimi, "Capillary effects during droplet impact on a solid surface," Phys. Fluids 8, 650 (1996).

${ }^{20}$ A. L. Yarin and D. A. Weiss, "Impact of drops on solid-surfaces-Self-similar capillary waves, and splashing as a new-type of kinematic discontinuity," J. Fluid Mech. 283, 141 (1995).

${ }^{21}$ I. V. Roisman, E. Berberovic, and C. Tropea, "Inertia dominated drop collisions. I. On the universal flow in the lamella," Phys. Fluids 21, 052103 (2009).

22 J. Eggers, M. A. Fontelos, C. Josserand, and S. Zaleski, "Drop dynamics after impact on a solid wall: Theory and simulations," Phys. Fluids 22, 062101 (2010).

${ }^{23}$ E. Villermaux and B. Bossa, "Drop fragmentation on impact," J. Fluid Mech. 668, 412 (2011).

${ }^{24}$ I. V. Roisman, "Inertia dominated drop collisions. II. An analytical solution of the Navier-Stokes equations for a spreading viscous film," Phys. Fluids 21, 052104 (2009).

${ }^{25}$ I. V. Roisman, R. Rioboo, and C. Tropea, "Normal impact of a liquid drop on a dry surface: Model for spreading and receding," Proc. R. Soc. London, Ser. A 458, 1411 (2002).

${ }^{26}$ I. V. Roisman, C. Planchette, E. Lorenceau, and G. Brenn, "Binary collisions of drops of immiscible liquids," J. Fluid Mech. 690, 512 (2012).

${ }^{27}$ G. Castanet, P. Dunand, O. Caballina, and F. Lemoine, "High-speed shadow imagery to characterize the size and velocity of the secondary droplets produced by drop impacts onto a heated surface," Exp. Fluids 54, 17 (2013).

${ }^{28}$ D. B. Reid, "An algorithm for tracking multiple targets," IEEE Trans. Autom. Control 24, 843 (1979).

${ }^{29}$ M. Rein, "Phenomena of liquid drop impact on solid and liquid surfaces," Fluid Dyn. Res. 12, 61 (1993).

${ }^{30}$ R. Rioboo, M. Marengo, and C. Tropea, "Time evolution of liquid drop impact onto solid, dry surfaces," Exp. Fluids 33, 112 (2002).

${ }^{31}$ S. Bakshi, I. V. Roisman, and C. Tropea, "Investigations on the impact of a drop onto a small spherical target," Phys. Fluids 19, 032102 (2007).

${ }^{32}$ N. P. van Hinsberg, M. Budakli, S. Göhler, E. Berberović, I. V. Roisman, T. Gambaryan-Roisman, C. Tropea, and P. Stephan, "Dynamics of the cavity and the surface film for impingements of single drops on liquid films of various thicknesses," J. Colloid Interface Sci. 350, 336 (2010).

${ }^{33}$ H. Lastakowski, F. Boyer, A. L. Biance, C. Pirat, and C. Ybert, "Bridging local to global dynamics of drop impact onto solid substrates," J. Fluid Mech. 747, 103 (2014).

${ }^{34}$ F. E. C. Culick, “Comments on a ruptured soap film,” J. Appl. Phys. 31, 1128 (1960).

${ }^{35}$ Glycerine Producers' Association, Physical Properties of Glycerine and Its Solutions (Glycerine Producers Association, 1963).

${ }^{36}$ G. Castanet, T. Liénart, and F. Lemoine, "Dynamics and temperature of droplets impacting onto a heated wall,” Int. J. Heat Mass Transfer 52, 679 (2009).

${ }^{37}$ P. Dunand, G. Castanet, and F. Lemoine, "A two-color planar LIF technique to map the temperature of droplets impinging onto a heated wall," Exp. Fluids 52, 843 (2012).

${ }^{38}$ P. Dunand, G. Castanet, M. Gradeck, D. Maillet, and F. Lemoine, "Energy balance of droplets impinging onto a wall heated above the Leidenfrost temperature," Int. J. Heat Fluid Flow 44, 170 (2013).

${ }^{39}$ H. Chaves, A. M. Kubitzek, and F. Obermeier, "Dynamic processes occurring during the spreading of thin liquid films produced by drop impact on hot walls," Int. J. Heat Fluid Flow 20, 470 (1999).

${ }^{40}$ D. Quéré, "Leidenfrost dynamics," Annu. Rev. Fluid Mech. 45, 197 (2013).

${ }^{41}$ R. C. A. van der Veen, T. Tran, D. Lohse, and C. Sun, "Direct measurements of air layer profiles under impacting droplets using high-speed color interferometry," Phys. Rev. E 85, 026315 (2012).

42 A. L. Biance, C. Clanet, and D. Quere, "Leidenfrost drops," Phys. Fluids 15, 1632 (2003).

${ }^{43}$ Y. Pomeau, M. Le Berre, F. Celestini, and T. Frisch, "The Leidenfrost effect: From quasi-spherical droplets to puddles," C. R. Mec. 340, 867 (2012).

${ }^{44}$ B. Sobac, A. Rednikov, S. Dorbolo, and P. Colinet, "Leidenfrost effect: Accurate drop shape modeling and refined scaling laws," Phys. Rev. E 90, 053011 (2014).

${ }^{45}$ A. H. Dawi, S. Herbert, I. V. Roisman, T. Gambaryan-Roisman, P. Stephan, and C. Tropea, "Numerical investigation of drop impact onto hot surfaces," in 25th European Conference on Liquid Atomization and Spray Systems, Chania, Crete, September 1-4, 2013. 